University of South Florida

DIGITAL COMMONS

Digital Commons @ University of

@ UNIVERSITY OF SOUTH FLORIDA

South Florida

6-1997

\title{
The Zonal Momentum Balance of the Equatorial Undercurrent in the Central Pacific
}

L. Qiao

University of South Florida

Robert $\mathrm{H}$. Weisberg

University of South Florida, weisberg@marine.usf.edu

Follow this and additional works at: https://digitalcommons.usf.edu/msc_facpub

Part of the Marine Biology Commons

\section{Scholar Commons Citation}

Qiao, L. and Weisberg, Robert H., "The Zonal Momentum Balance of the Equatorial Undercurrent in the Central Pacific" (1997). Marine Science Faculty Publications. 147.

https://digitalcommons.usf.edu/msc_facpub/147

This Article is brought to you for free and open access by the College of Marine Science at Digital Commons @ University of South Florida. It has been accepted for inclusion in Marine Science Faculty Publications by an authorized administrator of Digital Commons @ University of South Florida. For more information, please contact digitalcommons@usf.edu. 


\title{
The Zonal Momentum Balance of the Equatorial Undercurrent in the Central Pacific
}

\author{
L. QIAO AND R. H. WeISBERG \\ Department of Marine Science, University of South Florida, St. Petersburg, Florida
}

(Manuscript received 29 February 1996, in final form 25 November 1996)

\begin{abstract}
Current velocity data from an array of subsurface moorings deployed during the Tropical Instability Wave Experiment from May 1990 to June 1991 are used to diagnose the upper-ocean zonal momentum balance at $0^{\circ}$, $140^{\circ} \mathrm{W}$. The flow field and associated zonal momentum flux divergence are fully three-dimensional over the upper $250 \mathrm{~m}$, consistent with the earliest descriptions and theoretical ideas of the Equatorial Undercurrent (EUC). Estimates of the vertical stress divergence show dynamical flow regimes that change between the surface and the base of the EUC, being essentially linear (modified by nonlinearity) near the surface, weakly nonlinear at the EUC core, and fully nonlinear below the core. The vertical stress divergence is much larger over the lower portion of the EUC than previously reported, but this is consistent with the observed downstream deceleration of the EUC and the idea that vertical mixing is important in maintaining the thermostad. Nonlinearity becomes increasingly important with decreasing frequency, but tends to cancel upon vertical integration.
\end{abstract}

\section{Introduction}

The discovery by Cromwell et al. (1954) of a very swift, subsurface current, flowing eastward on the equator in opposition to the winds, initiated an ongoing dialog on the dynamics of this remarkable Equatorial Undercurrent (EUC). Early descriptive studies by Knauss (1960, 1966) showed the EUC to be continuous along, symmetric about, and tightly confined to the equator with transports comparable to other major ocean currents. Early theoretical studies, beginning with the nonlinear, inertial jet arguments of Fofonoff and Montgomery (1955) and the linear, frictional arguments of Arthur (1960) and Stommel (1960), were followed by numerous articles combining these arguments into more complete theories. Central to all of these is the three-dimensionality of the flow field, driven by a depth-dependent zonal pressure gradient (ZPG) whose vertical integral tends to balance a westward surface wind stress. This three-dimensionality is what makes the circulation so important to contemporary climate-related studies, because it largely determines the equatorial sea surface temperature distribution. But, it is also what makes a quantitative understanding of the EUC so difficult since it requires resolving the circulation's divergence.

In May 1990, an array of five subsurface acoustic Doppler current profiling moorings was deployed about $0^{\circ}, 140^{\circ} \mathrm{W}$ for 13 months as part of the Tropical Oceans

Corresponding author address: Dr. Robert H. Weisberg, Department of Marine Science, University of South Florida, 140 Seventh Avenue South, St. Petersburg, FL 33701-5016.

E-mail: weisberg@marine.usf.edu
Global Atmosphere (TOGA) Tropical Instability Wave Experiment (TIWE). The array provides estimates of the vertical circulation (Weisberg and Qiao 1996, unpublished manuscript) and thus a three-dimensional view of the flow field. The present paper uses these data to estimate the zonal momentum flux divergence and, combined with other data from the TOGA-Tropical Atmosphere Ocean (TOGA-TAO) array, to diagnose the upperocean zonal momentum balance. Section 2 reviews previous work on this topic. Section 3 describes the data and methods. Section 4 attempts a quantitative diagnosis of the record-length averaged, depth-dependent zonal momentum balance and provides a description of how the dynamics change between the surface and the base of the EUC. Section 5 offers a more qualitative (owing to data limitations) view of the time-dependent variations. These findings are then summarized and discussed in section 6 .

\section{Background}

Knauss (1960,1966) gives a comprehensive description of the EUC along with dynamical inferences. The flow is three-dimensional with a meridional convergence upon the EUC core compensated by a vertical divergence away from the core. Intense vertical mixing is surmised on the equator to account for both the observed material property distributions in the meridional plane and the approximate geostrophic balance (meridionally) found for the near-equator zonal currents. Meridional convergence upon the EUC core is the essential element in Fofonoff and Montgomery (1955) where the speed of the EUC is accounted for by conservation of 
absolute vorticity. This convergence is attributed to an eastward directed ZPG force owing to a westward wind stress over a bounded basin. Recognizing that the effects of the wind-induced surface stress may extend vertically over the same region for which the ZPG is dynamically significant, Arthur (1960) calculated a velocity profile on the equator from the balance between the vertical stress divergence and the ZPG. Thus, the EUC core occurs where the stress is zero and the stress divergence crosses zero together with the ZPG. Charney (1960) and Charney and Spiegel (1971) combined these inertial and viscous effects in a constant density EUC model and found that the relative importance of these terms greatly affects the resulting three-dimensional flow field.

Theory and observations confirm that the zonal momentum balance on the equator must entail convergences of momentum flux and stress along with a ZPG, but further advances have been hampered by data limitations. Simultaneous data have been unavailable for estimating these constituents, leaving uncertainty in the zonal momentum balance for both analytical and numerical model results.

In a diagnostic study, Bryden and Brady (1985) used historical hydrographic data for a box bounded by $5^{\circ} \mathrm{S}$ and $5^{\circ} \mathrm{N}, 150^{\circ} \mathrm{W}$ and $110^{\circ} \mathrm{W}$, and $500 \mathrm{db}$ and the surface. The mean horizontal pressure gradient was referenced to $500 \mathrm{db}$; the horizontal velocity components were estimated from the horizontal pressure gradient components and the climatological wind stress using geostrophic and Ekman assumptions, and the vertical velocity component $(\mathrm{w})$ was then calculated by mass conservation. The ZPG decreased monotonically to zero between the surface and the lower portion of the EUC and was slightly westward below. Its vertical integral balanced the surface wind stress to within about $80 \%$. The vertical integral of the nonlinear accelerations overcompensated the surface wind stress/ZPG imbalance, implying a significant stress (and stress divergence) at least to the base of the EUC. On the equator upwelling was found above $180 \mathrm{db}$ with smaller downwelling below, and the flow was described as being primarily along isopycnals.

The $w$ profile of Bryden and Brady (1985) has been used in subsequent studies for estimating the vertical advection of eastward momentum. An example is given by Wilson and Leetmaa (1988), employing data from several shipboard velocity profile and hydrographic surveys on the equator roughly between $150^{\circ} \mathrm{W}$ and $90^{\circ} \mathrm{W}$. The time-dependent variations for the estimated terms were found to be as large as their means. Upon vertical integration the ZPG closely balanced the wind stress, but the lack of data on individual terms precluded analyses on the vertical profiles. However, their estimate of vertical eddy viscosity showed large values below the EUC core. Another example is that of McPhaden and Taft (1988), wherein the zonal momentum balance is studied between $140^{\circ} \mathrm{W}$ and $110^{\circ} \mathrm{W}$ using TOGA-TAO array moored current meter data. The vertical profiles of the ZPG and the estimated nonlinear accelerations were very similar to those of Bryden and Brady (1985), as was the imbalance in the integrated ZPG and the surface stress. The mean accelerations tended to oppose each other, suggesting that nonlinearity redistributes momentum vertically in the zonal plane within the upper $250 \mathrm{~m}$. Assuming a small, constant vertical eddy viscosity coefficient $A_{v}=1 \times 10^{-4} \mathrm{~m}^{2} \mathrm{~s}^{-1}$ (motivated by the microstructure measurements of Peters et al. 1988), the vertical stress at $250 \mathrm{~m}$ was calculated to be 100 times smaller than the surface stress. Thus, the vertically integrated imbalance was not resolved. A time-dependent analysis showed that on intraseasonal timescales the vertically integrated ZPG varied with the surface stress to within about the same imbalance as the mean.

In an attempt to resolve the role of turbulent stress divergence, Hebert et al. (1991) used shipboard microstructure and moored measurements collected between $140^{\circ} \mathrm{W}$ and $110^{\circ} \mathrm{W}$ in spring 1987 . With zonal advection being the only calculable nonlinear acceleration term, correspondences were not achieved between the estimated ZPG, acceleration, and turbulent stress divergence. It was suggested that annual averages are necessary for comparing estimates of turbulent stress divergence with the diagnostic calculation of Bryden and Brady (1985).

Turbulent stress divergence occurs over synoptic as well as microstructure and intermediate scales. At synoptic scales, the tropical instability waves are particularly important. For example, the horizontal Reynolds stress divergence on the equator in the central Pacific is a significant fraction of the wind-stress-induced body force (Hansen and Paul 1984), and similarly for the Atlantic (Weisberg and Weingartner 1988). Additional supporting evidence is found in Lukas (1987), Wilson and Leetmaa (1988), Bryden and Brady (1989), and Luther and Johnson (1990). However, even when averaging over a record length sufficient to include the synoptic scale (Johnson and Luther 1994), the role of the turbulent stress divergence on the equator has remained an unresolved issue.

In summary, the available datasets show an approximate balance on the equator between the vertically integrated ZPG force and the surface stress. Estimates of the nonlinear acceleration terms suggest that these tend to cancel, but not completely. Since the nonlinear accelerations may be important in adjusting the flow field to external forcing, their resolution is necessary for determining the vertical distribution of stress divergence and hence an improved understanding of the equatorial currents' zonal momentum balance.

\section{Data and methods}

The TIWE equatorial moorings, designated TIW1TIW5, were deployed in a diamond shaped array centered upon $0^{\circ}, 140^{\circ} \mathrm{W}$ (Fig. 1). Hourly velocity profiles were sampled by RD-Instruments $150-\mathrm{kHz}$ acoustic 


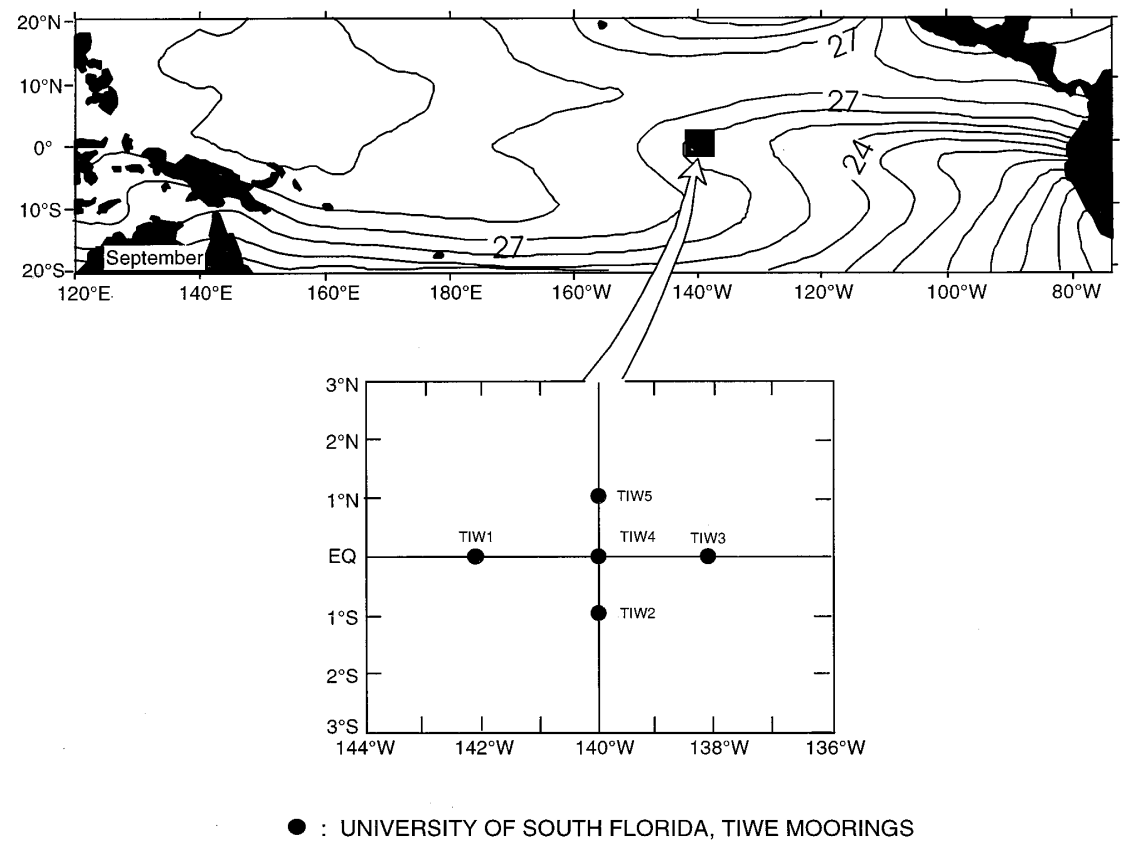

FIG. 1. The location of the TIWE equatorial array in relation to the tropical Pacific Ocean's climatological SST distribution for September (Courtesy of M. McCarty and M. McPhaden, NOAA/PMEL).

Doppler current profilers (ADCP) with $20^{\circ}$ transducer configuration. Mooring performance and data editing procedures are given in Weisberg et al. (1991). With ambient sound speed correction, the instruments nominally sampled at $9-\mathrm{m}$ vertical intervals. Hourly velocity components resampled at $10-\mathrm{m}$ intervals between 250 $\mathrm{m}$ and the surface are used herein. Resampling was by linear interpolation between 250 and $30 \mathrm{~m}$ (the last bin unbiased by surface reflection) and by linear extrapolation over the upper $20 \mathrm{~m}$ using the 30-40 m shear. The analysis period is 12 May 1990 to 18 June 1991 and the mooring locations and nominal instrument depths are listed in Table 1.

The time and depth variations of the zonal $(u)$ and meridional $(v)$ velocity components at the five mooring locations are shown in Figs. 2 and 3, where the data here and in subsequent time series plots are low-pass

TABLE 1. TIWE equatorial array mooring positions and nominal instrument depths.

\begin{tabular}{ccc}
\hline \hline $\begin{array}{c}\text { Mooring } \\
\text { name }\end{array}$ & $\begin{array}{c}\text { Position } \\
\text { (lat/long) }\end{array}$ & $\begin{array}{c}\text { Instrument depth } \\
(\mathrm{m})\end{array}$ \\
\hline TIW1 & $0^{\circ} 01.4^{\prime} \mathrm{N}$ & 273.6 \\
& $141^{\circ} 50.6^{\prime} \mathrm{W}$ & \\
TIW2 & $0^{\circ} 57.8^{\prime} \mathrm{S}$ & 280.5 \\
& $139^{\circ} 57.5^{\prime} \mathrm{W}$ & \\
TIW3 & $0^{\circ} 02.4^{\prime} \mathrm{N}$ & 281.5 \\
& $137^{\circ} 57.7^{\prime} \mathrm{W}$ & \\
TIW4 & $0^{\circ} 03.2^{\prime} \mathrm{S}$ & 276.5 \\
& $140^{\circ} 08.4^{\prime} \mathrm{W}$ & \\
TIW5 & $1^{\circ} 01.5^{\prime} \mathrm{N}$ & 266.4 \\
& $139^{\circ} 57.4^{\prime} \mathrm{W}$ & \\
\hline
\end{tabular}

filtered to exclude oscillations at timescales shorter than 10 days. The $u$-component isotachs are representative of the zonally oriented equatorial currents reported on by numerous precedent studies. Observed is a highly variable, near-surface-confined South Equatorial Current (SEC) overriding the EUC whose high speed core is located within the thermocline (Fig. 6). The five locations show an EUC that is maximum on, and nearly symmetric about, the equator and a SEC that is maximum to the north of the equator. The primary variations in the EUC are both annual and intraseasonal. Annually, the depth of the EUC's high speed core varies with the thermocline with maximum EUC speed observed in July 1990 and April 1991 when the core was relatively shallow. In contrast, an intraseasonal maximum is observed in December 1990 when the core was relatively deep. In agreement with previous studies the SEC is most developed when the EUC is deepest, except when intervened upon by large intraseasonal events such as one in December 1990. When the EUC is shallow, the SEC is weak, and westward flow was generally absent on the equator from April to June 1991.

In contrast to $u$, the $v$ component consists of seasonally modulated, higher frequency oscillations. In particular, a series of regular, large amplitude oscillations are observed at all of the sample locations from August to December 1990. These are the tropical instability waves and a description of their kinematics during this time is given by Qiao and Weisberg (1995). The $v$ component oscillations are largest on the equator within the westward-flowing SEC, with amplitudes decreasing 

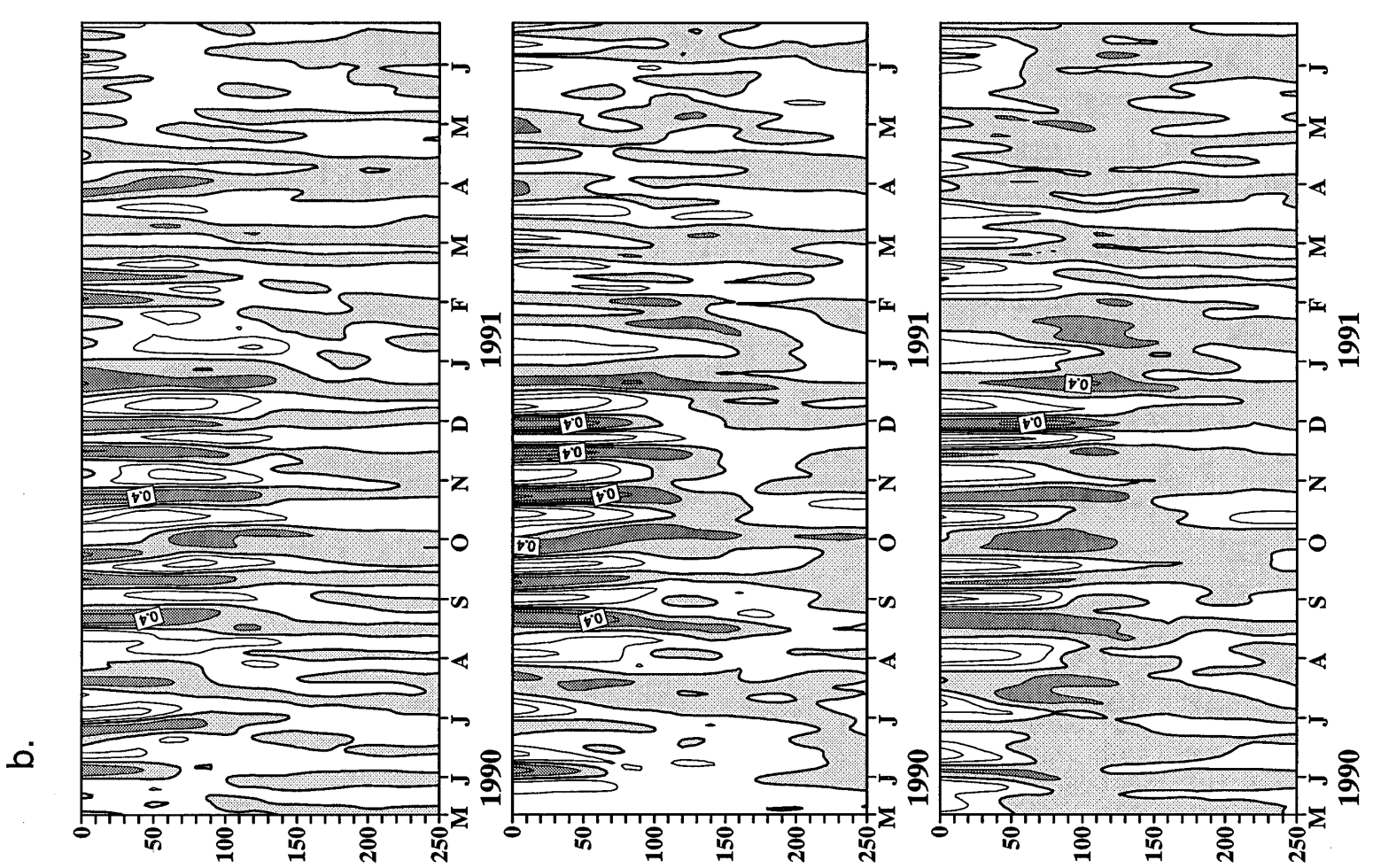

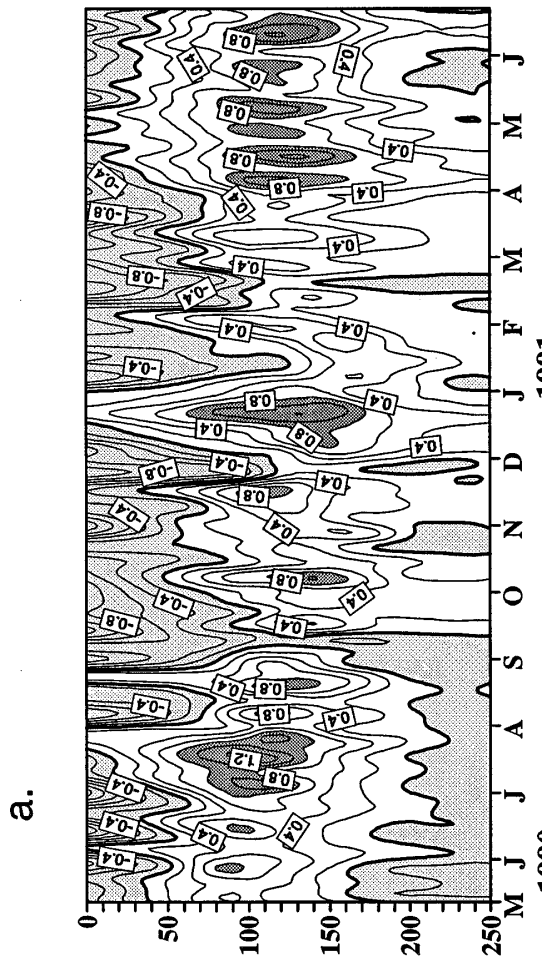

(ui) प1dә्व

$\mathbf{M}_{0} \mathbf{O b I}{ }^{6} \mathbf{N}_{\mathrm{o}} \mathrm{I}$

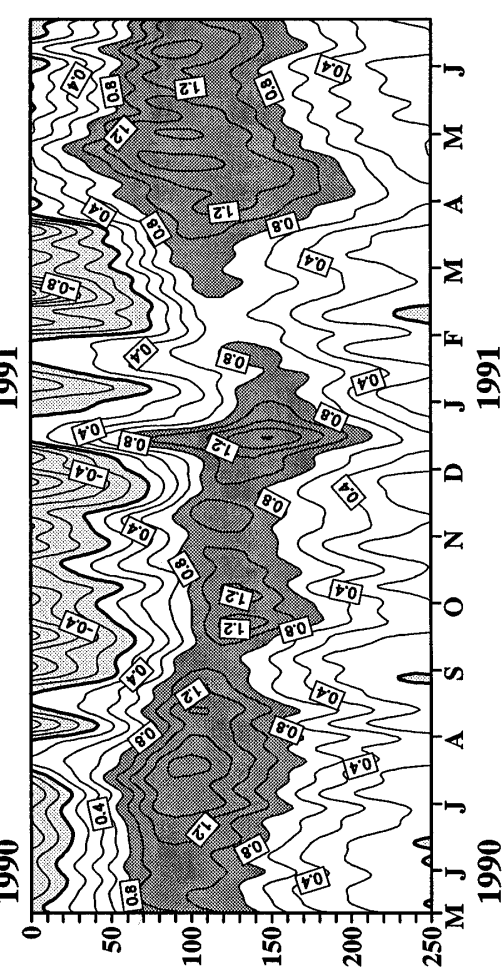

(ui) प1dәव

$\mathbf{M}_{0} 0$ t大 I ${ }^{6} 0$

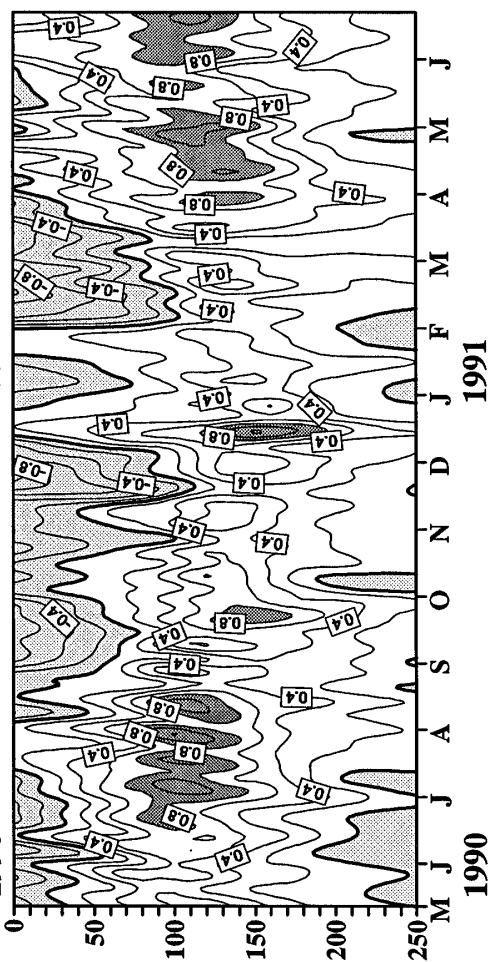

(ui) प1dә्व

$M_{0} 0$ t I ' $S_{0} I$

ป 웡

님웜

을

6.

믕 륳

흘

응

:

ఫี 

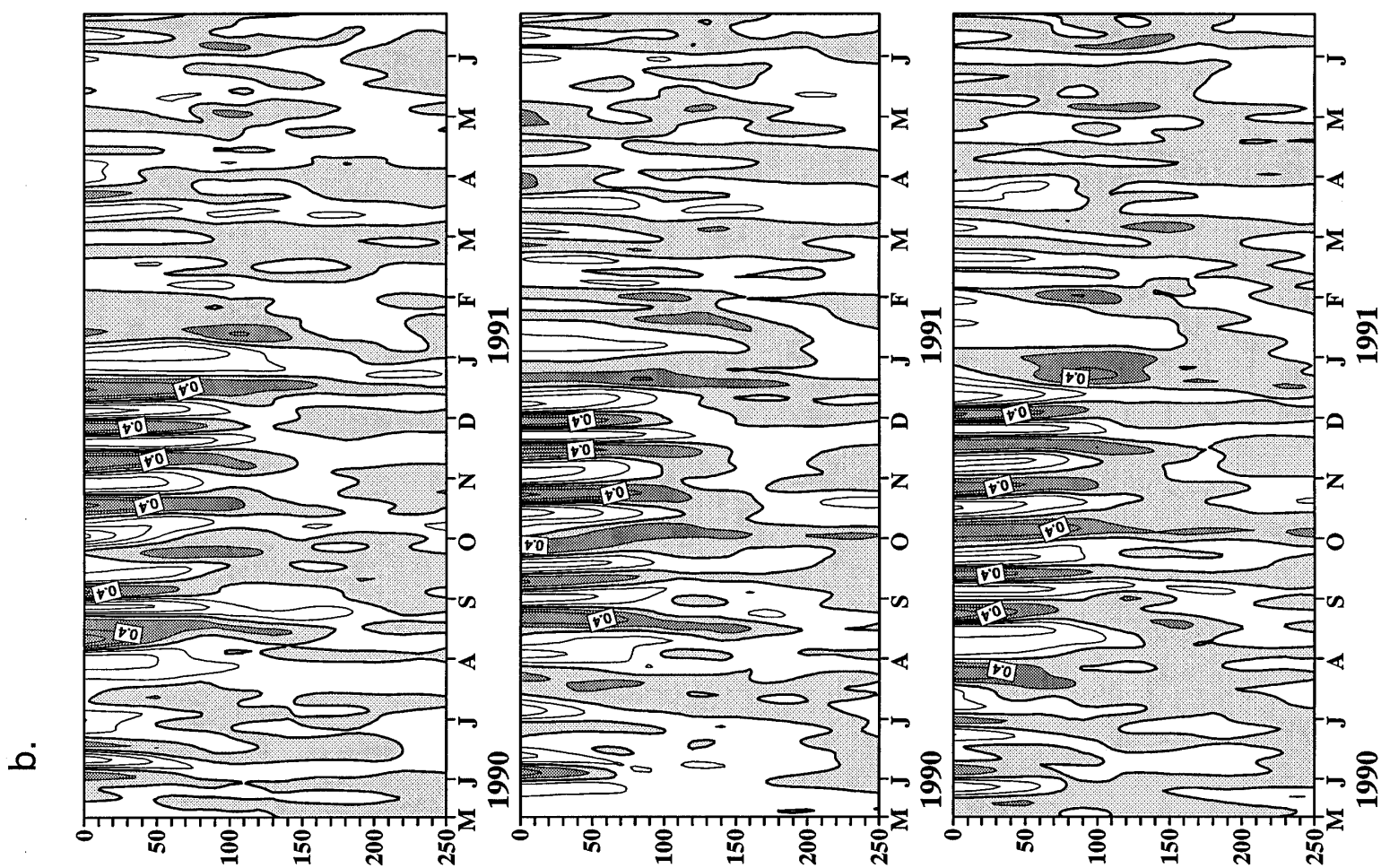

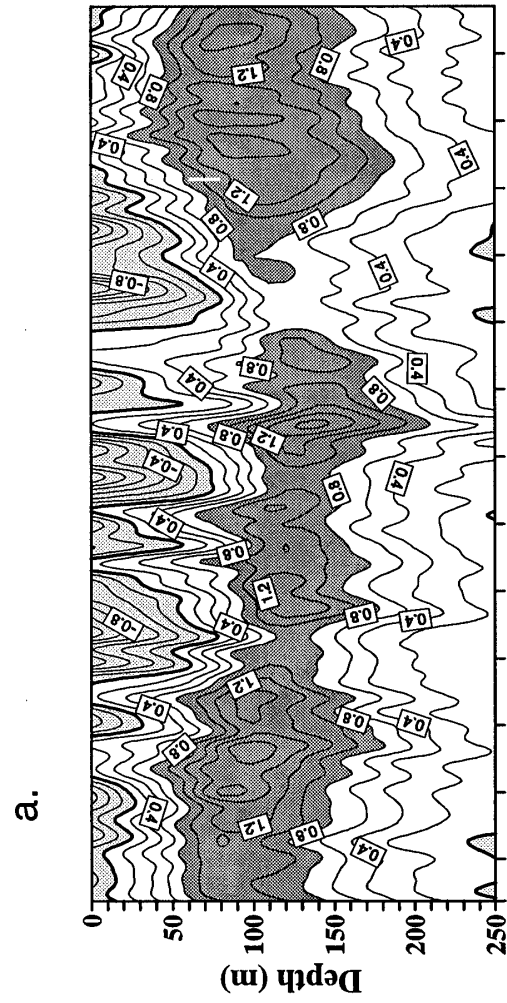

$M_{0} 8 E I$ '。O

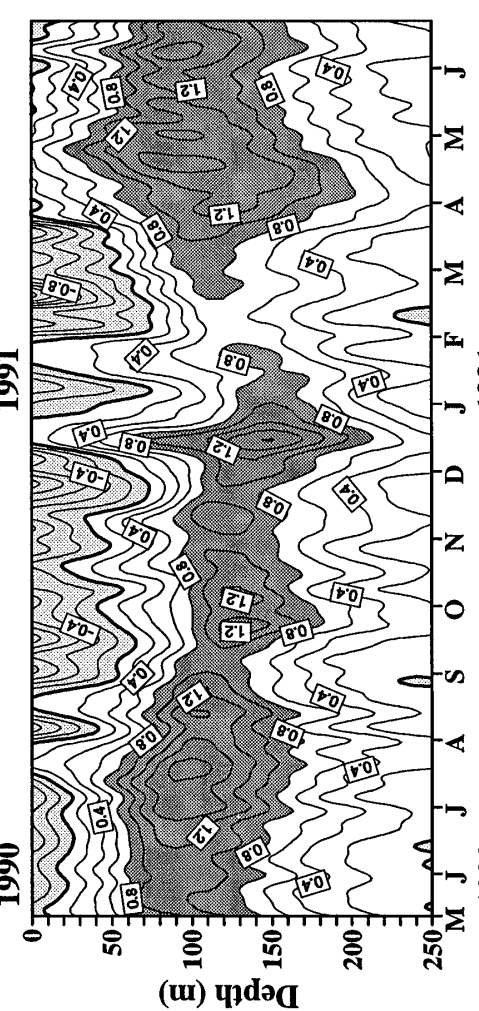

$\mathbf{M}_{0} 0$ OtI I'00

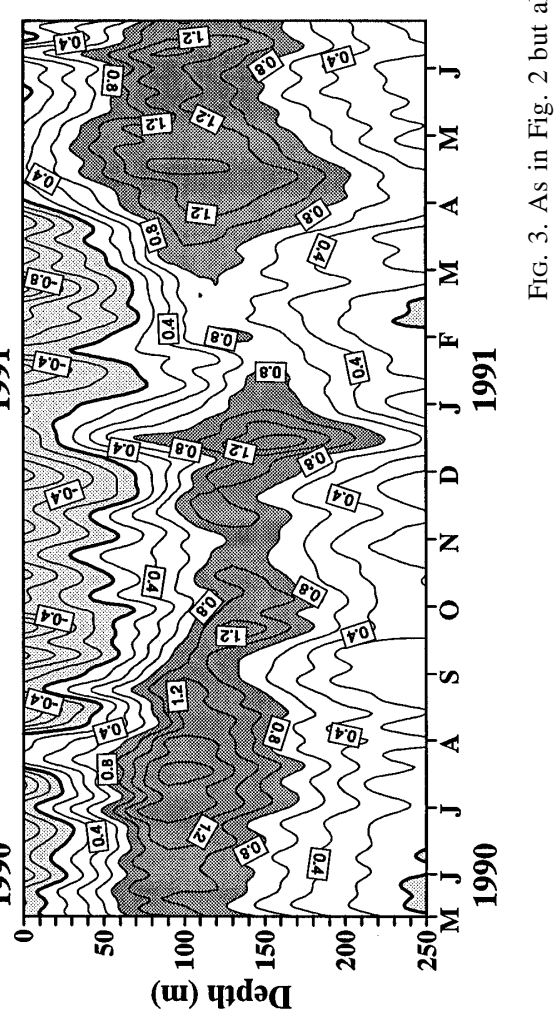

$M_{0} Z t I{ }^{\circ} 0$ 

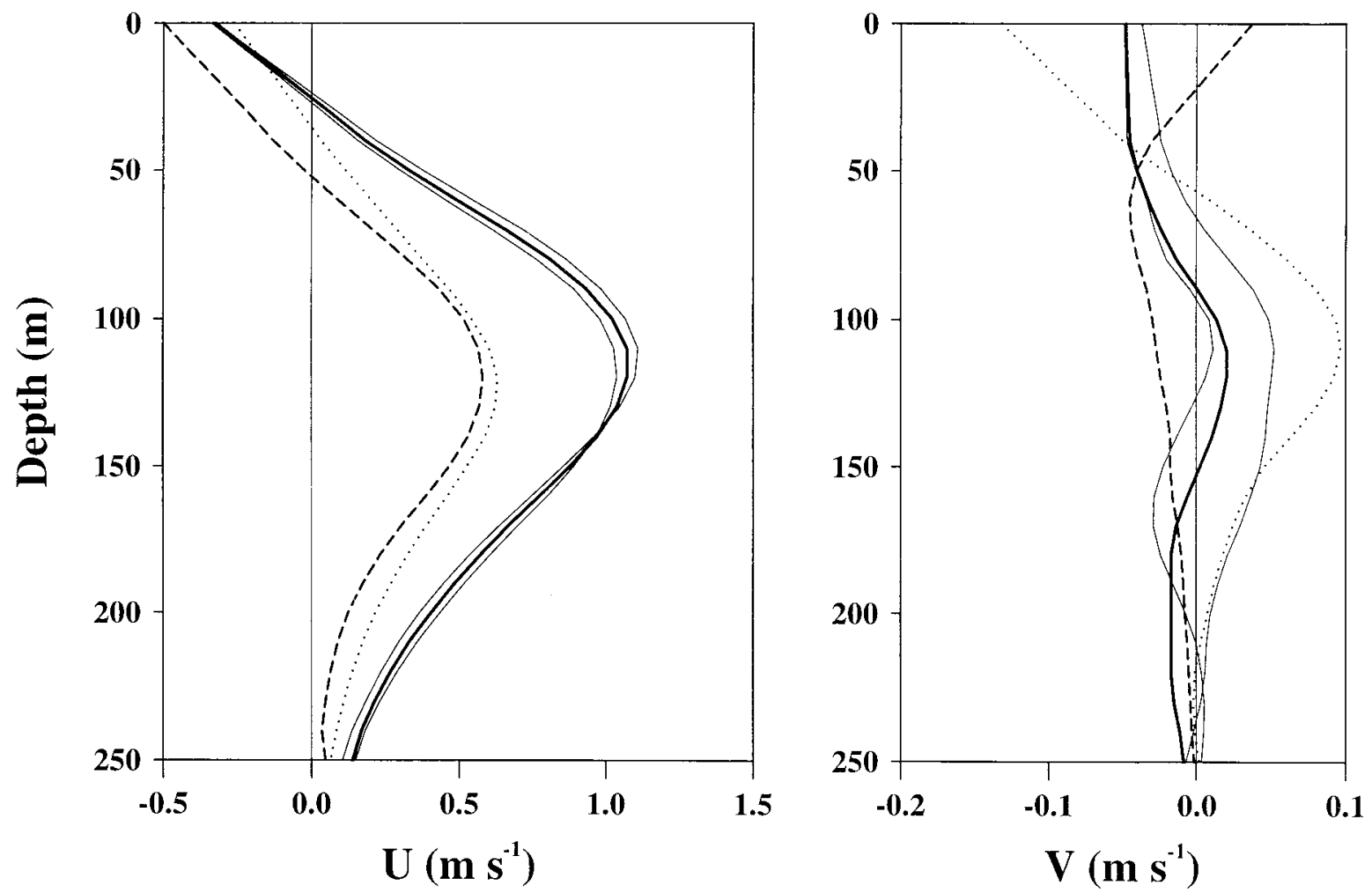

FIG. 4. Record-length-mean vertical profiles for the $u$ and $v$ components at the five mooring locations. The solid lines denote the three equator moorings (the thick one being $140^{\circ} \mathrm{W}$ ) and the dashed and dotted lines denote moorings north and south of the equator, respectively.

precipitously across the thermocline to relatively small values at the EUC core.

Mean horizontal velocity component vertical profiles are obtained by averaging over the 13-month record length (Fig. 4). For the $u$ component the three equatorial locations are similar, except that the speed at the EUC core increases downstream as the core depth shoals to the east. The core speeds at the two off-equator locations are nearly symmetric and about $60 \%$ of the values on the equator. The SEC is shallow at all locations but penetrates slightly deeper off the equator. For the $v$ component, the off-equator locations indicate antisymmetric behavior consistent with a surface Ekman divergence and a subsurface geostrophic convergence. The three locations on the equator all show similar vertical profiles intermediate between the off-equator ones so the mean meridional divergence is nearly symmetric about the equator. At $250 \mathrm{~m}$, or the base of the EUC, all of the $v$ component profiles approach zero.

Vertically integrating the continuity equation between the surface and depth $\mathrm{z}$ provides an estimate $\mathrm{w}$ :

$$
w(z)=w(0)+\int_{z}^{0}\left(\frac{\partial u}{\partial x}+\frac{\partial v}{\partial y}\right) d z,
$$

where $x, y$, and $z$ are positive to the east, north, and up, respectively. Central differences are used for the zonal and meridional derivatives followed by vertical integration using trapezoidal rule such that

$$
w(z)=w(0)+\sum_{z}^{0}\left(\frac{u_{3}-u_{1}}{x_{3}-x_{1}}+\frac{v_{5}-v_{2}}{y_{5}-y_{2}}\right) \Delta z,
$$

where the subscripts denote the station locations and $\Delta \mathrm{z}$ is $10 \mathrm{~m}$. A rigid-lid approximation is used for $\mathrm{w}(0)$, which is correct to a factor of about $10^{-2}$ for the synoptic or longer timescales considered.

The resulting (low-pass filtered) $w$ at $0^{\circ}, 140^{\circ} \mathrm{W}$ is shown as a function of time and depth in Fig. 5 and the corresponding record-length-averaged vertical profiles for $u, v$, and $w$ are given in Fig. 6. The low-frequency variations in $\mathrm{w}$ are of order $10^{-5}$ to $10^{-4} \mathrm{~m} \mathrm{~s}^{-1}$. The mean $w$ profile shows maximum upwelling of about 2.3 $\times 10^{-5} \mathrm{~m} \mathrm{~s}^{-1}$ at $60-\mathrm{m}$ depth and a zero crossing at 140 $m$ just below the EUC core. The lower portion of the EUC is thus a region of downwelling on average. The fluctuations in $w$ are a factor of 5-10 larger than the mean and these may be of the same sign over the entire region of the water column sampled. A specific example is the intraseasonal event in December 1990, when downwelling is observed at the leading edge of an eastward momentum pulse followed by upwelling at the trailing edge, consistent with the passage of an equatorial Kelvin wave. A more detailed discussion of $w$, 


\section{$\mathrm{W}\left(10^{-5} \mathrm{~m} \mathrm{~s}^{-1}\right)$}

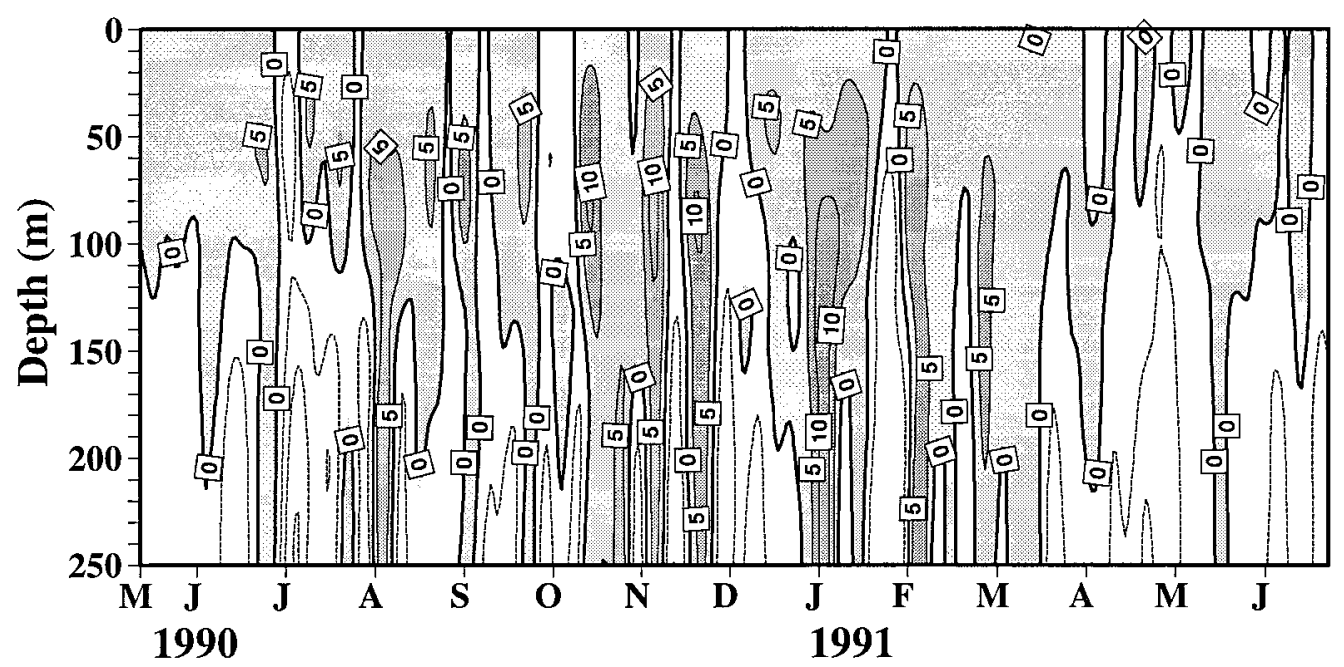

FIG. 5. The $w$ component as a function of depth and time estimated at $0^{\circ}, 140^{\circ} \mathrm{W}$. Upwelling is denoted by stippling and the contour interval is $5 \times 10^{-5} \mathrm{~m} \mathrm{~s}^{-1}$. The time series have been low-pass filtered to exclude fluctuations at timescales shorter than 10 days.

including error estimates, comparisons with previous estimates, and implications regarding the upper-ocean heat balance are in preparation (Weisberg and Qiao 1996, unpublished manuscript). Here we note that the mean $w$ profile is robust with regard to random errors,

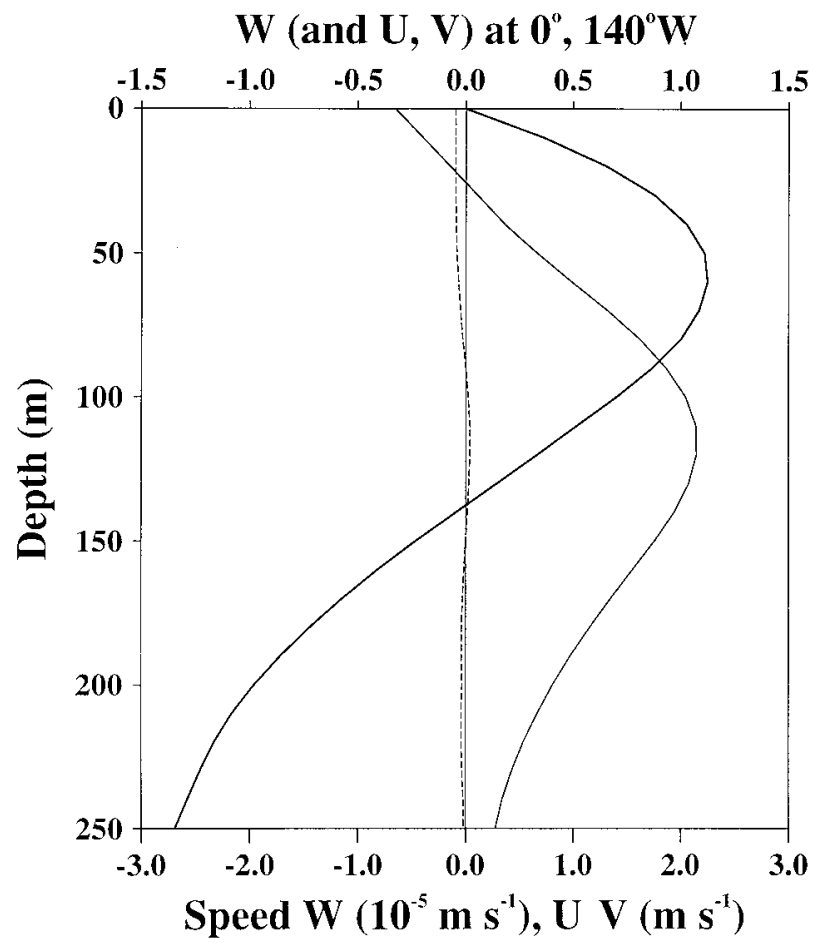

FIG. 6. The record-length-mean vertical profiles of the $u$ (thin solid), $v$ (dashed), and $w$ (thick solid) components at $0^{\circ}, 140^{\circ} \mathrm{W}$ that the potential for systematic errors owing to compass calibration when vertically integrated to $250 \mathrm{~m}$ are an order of magnitude less than the estimated mean $w$ there, and that kinematic, dynamic, and thermodynamic consistencies argue against large finite differencing errors. This is important since the downwelling estimated below the EUC core (with magnitude similar to that of the upwelling above the core) is an essential element in the momentum arguments that follow.

Temperature and wind data used for estimating the ZPG and the surface wind stress, respectively, are from the TOGA-TAO array. Figure 7 shows (low-pass filtered) temperature as a function of time and depth on the equator at $170^{\circ} \mathrm{W}, 140^{\circ} \mathrm{W}$, and $125^{\circ} \mathrm{W}$. Consistent with previous observations, the thermocline slopes up to the east giving rise to the depth-dependent ZPG. Superimposed on the isotherms at $170^{\circ}$ and $140^{\circ} \mathrm{W}$ is the EUC core depth, which coincides and shoals with the thermocline. Also, the depth of penetration for the westward SEC decreases eastward as the mixed layer becomes shallower.

\section{The mean zonal momentum balance}

Beginning with the zonal momentum balance in the form

$$
\begin{aligned}
\frac{\partial u}{\partial t}+u \frac{\partial u}{\partial x}+v \frac{\partial u}{\partial y}+w \frac{\partial u}{\partial z}-f v= & -\frac{1}{\rho} \frac{\partial P}{\partial x}+\frac{\partial}{\partial z}\left(\nu \frac{\partial u}{\partial z}\right) \\
& +\nabla \cdot(\nu \nabla u),
\end{aligned}
$$

where $f$ is the Coriolis parameter, $p$ is pressure, $\rho$ is density, $\nu$ is viscosity, and $\boldsymbol{\nabla}$ is a horizontal gradient 

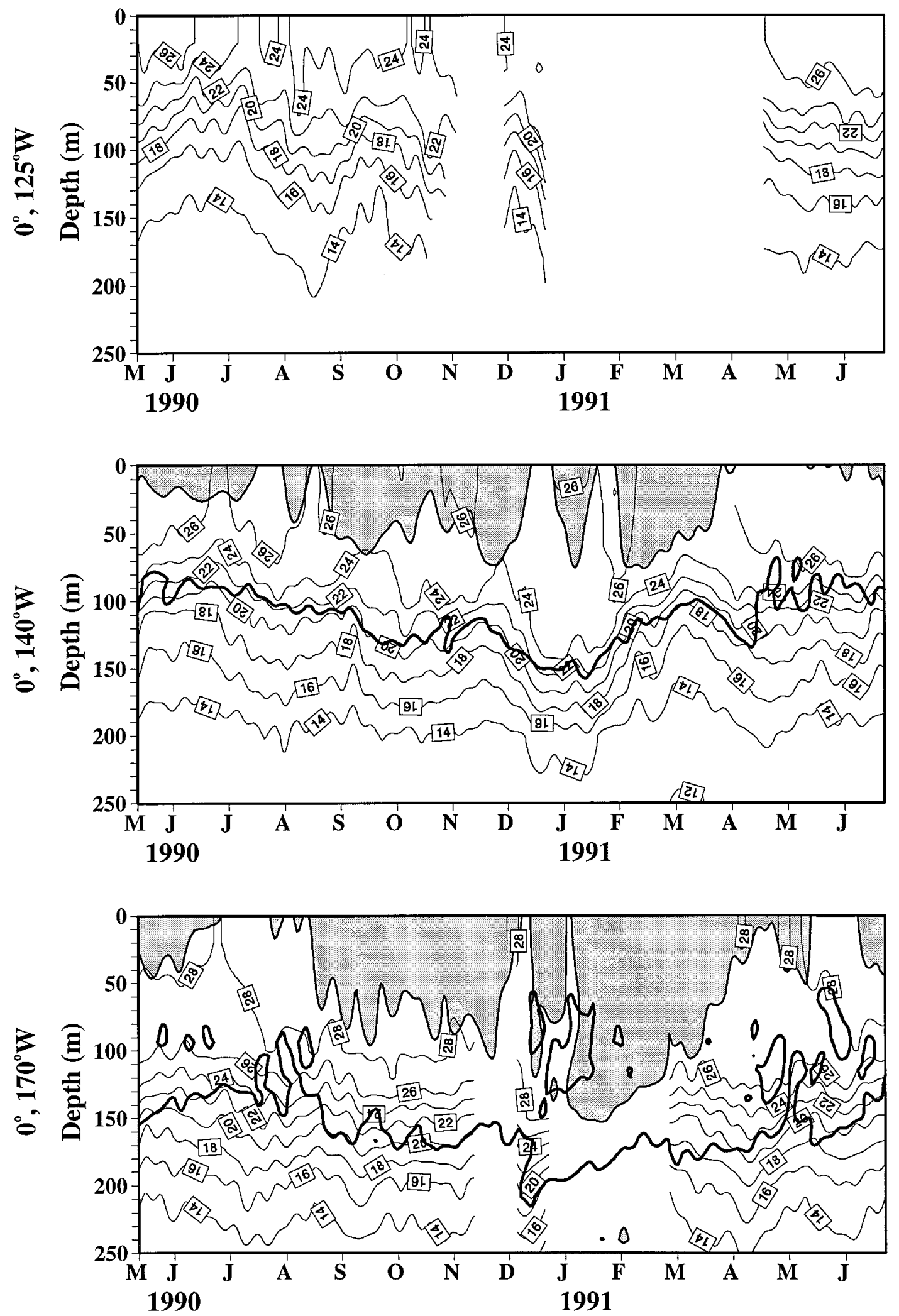

FIG. 7. Isotherm depths as a function of time from moorings at $0^{\circ}, 125^{\circ} \mathrm{W} ; 0^{\circ}, 140^{\circ} \mathrm{W}$; and $0^{\circ}, 170^{\circ} \mathrm{W}$ (from the TOGATAO array courtesy of M. McPhaden, NOAA/PMEL). Superimposed at $0^{\circ}, 170^{\circ} \mathrm{W}$ and $0^{\circ}, 140^{\circ} \mathrm{W}$ are the EUC core depths defined by $\partial u / \partial z=0$ and the westward-flowing SEC regions denoted by stippling. 
operator, and then averaging over the record length, gives the mean equation

$$
\begin{aligned}
\left\langle u \frac{\partial u}{\partial x}\right\rangle+\left\langle v \frac{\partial u}{\partial y}\right\rangle+\left\langle w \frac{\partial u}{\partial z}\right\rangle-f V= & -\left\langle\frac{1}{\rho} \frac{\partial P}{\partial x}\right\rangle+\frac{\partial}{\partial z}\left(A_{v} \frac{\partial U}{\partial z}\right) \\
& +\nabla \cdot\left(A_{h} \nabla U\right)
\end{aligned}
$$

where the angle brackets denote the record-length average, capitalized variables are the record-length means, and $A_{\mathrm{v}}$ and $A_{\mathrm{h}}$ are vertical and horizontal eddy viscosity coefficients dependent upon the averaging interval. Each of the nonlinear acceleration terms may be decomposed into mean circulation and Reynolds stress terms as

$$
\begin{aligned}
& \left\langle u \frac{\partial u}{\partial x}\right\rangle=U \frac{\partial U}{\partial x}+\left\langle u^{\prime} \frac{\partial u^{\prime}}{\partial x}\right\rangle \\
& \left\langle\begin{array}{l}
\left.v \frac{\partial u}{\partial y}\right\rangle
\end{array}\right\rangle=V \frac{\partial U}{\partial y}+\left\langle v^{\prime} \frac{\partial u^{\prime}}{\partial y}\right\rangle \\
& \left\langle w \frac{\partial u}{\partial z}\right\rangle=W \frac{\partial U}{\partial z}+\left\langle w^{\prime} \frac{\partial u^{\prime}}{\partial z}\right\rangle,
\end{aligned}
$$

where the primes denote (hourly) fluctuations about the record-length means.

The nonlinear acceleration terms in the above formulation are expressed using an advective scheme. Alternatively, using the continuity equation, these may be expressed using a flux divergence scheme, wherein their record-length averages are

$$
\begin{aligned}
\frac{\partial\langle u u\rangle}{\partial x}+\frac{\partial\langle u v\rangle}{\partial y}+\frac{\partial\langle u w\rangle}{\partial z}= & \frac{\partial U U}{\partial x}+\frac{\partial U V}{\partial y}+\frac{\partial U W}{\partial z} \\
& +\frac{\partial\left\langle u^{\prime} u^{\prime}\right\rangle}{\partial x}+\frac{\partial\left\langle u^{\prime} v^{\prime}\right\rangle}{\partial y} \\
& +\frac{\partial\left\langle u^{\prime} w^{\prime}\right\rangle}{\partial z}
\end{aligned}
$$

In either case, central differences are used to estimate the horizontal derivatives; thus, for the advective and flux divergence schemes the acceleration terms become

$$
\begin{aligned}
& p \frac{\partial q}{\partial x}=p_{4} \frac{q_{3}-q_{1}}{x_{3}-x_{1}} \quad \text { or } \quad \frac{\partial p q}{\partial x}=\frac{p_{3} q_{3}-p_{1} q_{1}}{x_{3}-x_{1}} \\
& p \frac{\partial q}{\partial y}=p_{4} \frac{q_{5}-q_{2}}{y_{5}-y_{2}} \quad \text { or } \quad \frac{\partial p q}{\partial y}=\frac{p_{5} q_{5}-p_{2} q_{2}}{y_{5}-y_{2}},
\end{aligned}
$$

where $p$ and $q$ represent the appropriate horizontal velocity components and subscripts denote horizontal locations (Table 1). The vertical derivatives are calculated by forward differencing consistent with the $w$ estimation.

Given the mean circulation's symmetry about the equator (that results in both $\langle v\rangle$ and $\langle\partial u / \partial y\rangle$ being appproximately zero on the equator) the flux divergence scheme is necessary to reveal the fully three-dimen- sional nature of the nonlinear zonal momentum flux. This same symmetry, however, biases the flux divergence scheme relative to the advective scheme. For these reasons the flux divergence scheme results are presented first to develop the three-dimensionality of the zonal momentum flux. This is followed by the advective scheme results, and the bias for the flux divergence scheme is accounted for in appendix B. Estimation is then made of the ZPG and the zonal wind stress $\left(\tau_{0}^{x}\right)$ and the results are combined into vertically integrated and pointwise momentum balances from which the vertical stress divergence follows as a residual. Integrating the vertical stress divergence then results in vertical profiles of stress and $A_{v}$.

\section{a. Material acceleration by flux divergence formulation}

Vertical profiles of the nonlinear terms of (5) are shown in Fig. 8. They are arranged such that the left, central, and right columns provide the divergence of means, the divergence of the Reynolds fluxes, and the divergence of their sums, respectively, and the rows from top to bottom provide the zonal, meridional, and the vertical derivatives comprising these mean divergences. Standard deviations due to random variations (derived in appendix A) are indicated by dashed lines. Relative to these, all of the estimated nonlinear terms are significantly different from zero at most depths (excepting $\left.\partial\left\langle u^{\prime} v^{\prime}\right\rangle / \partial y\right)$ and the shapes and zero-crossings of the vertical profiles are robust.

The mean zonal derivative $\partial U U / \partial x$ is nearly zero above $40 \mathrm{~m}$, positive from $40 \mathrm{~m}$ to $140 \mathrm{~m}$, and negative below $140 \mathrm{~m}$. The zero-crossing at $140 \mathrm{~m}$ (about $30 \mathrm{~m}$ below the EUC core) is consistent with the fact that the flow within the EUC core accelerates as the core shoals downstream (Fig. 4) at this location. This finding of a zero-crossing below the core differs from the Bryden and Brady (1985) and McPhaden and Taft (1988) findings of zero-crossings either at or above the core. Zonal finite differences for these previous studies, however, were over $30^{\circ}$ of longitude, a distance over which the vertical position of the core changed on a scale comparable to the measurement's vertical resolution. With higher zonal and vertical resolution $\partial U U / \partial x$ is observed to be maximum and positive at the EUC core and negative below the EUC core.

The vertical profile of the mean meridional derivative $\partial U V / \partial y$ is negative with largest magnitudes at the surface and at the EUC core. At the surface, this is due to the Ekman divergence of westward momentum away from equator and at the EUC core, this is due to the geostrophic convergence of eastward momentum onto the equator. The minimum at $40 \mathrm{~m}$ marks the transition between Ekman divergence and geostrophic convergence dominance, and $\partial U V / \partial y$ then approaches zero again below $220 \mathrm{~m}$ as the geostrophic convergence goes to zero at the base of the EUC. 

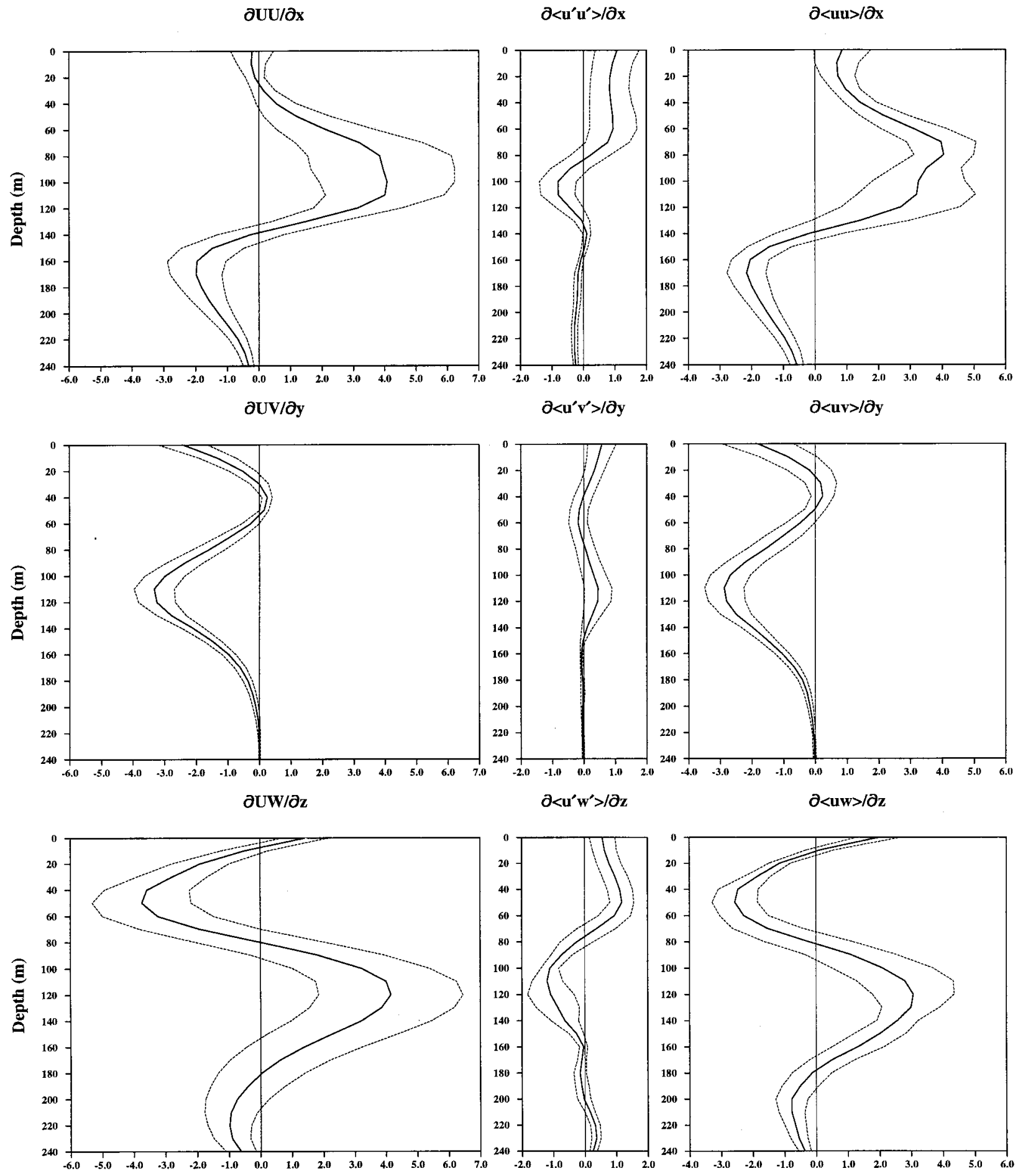

Acceleration $\left(10^{-5} \mathrm{~cm} \mathrm{~s}^{-2}\right)$

Acceleration $\left(10^{-5} \mathrm{~cm} \mathrm{~s}^{-2}\right)$

Acceleration $\left(10^{-5} \mathrm{~cm} \mathrm{~s}^{-2}\right)$

FIG. 8. Vertical profiles of the individual constituents making up the record-length-mean zonal momentum flux divergence estimated about $0^{\circ}, 140^{\circ} \mathrm{W}$. The dashed lines represent standard deviations by random fluctuations (appendix A). 
The vertical profile of the mean vertical derivative $\partial U W / \partial z$ varies in opposition to that of $\partial U V / \partial y$ with relative maxima and minima of opposite sign occurring at the same depths. In particular, within the EUC core, the divergence of eastward momentum by the vertical circulation nearly cancels the convergence of eastward momentum by the meridional circulation. Moreover, all of these mean derivative terms are of the same magnitude, showing that the mean divergence about the equator of the mean eastward momentum is fully three-dimensional.

The mean divergences of the zonal momentum flux owing to the Reynolds fluxes (the three central panels in Fig. 8) are generally smaller than, and tend to oppose, their corresponding mean circulation terms (the three left panels of Fig. 8). Intuitively, the Reynolds fluxes act to smooth the mean gradients. An exception is the near-surface behavior of $\partial\left\langle u^{\prime} u^{\prime}\right\rangle / \partial x$, which adds to $\partial U U /$ $\partial x$ between 30 and $90 \mathrm{~m}$ and below $150 \mathrm{~m}$. Also, $\partial\left\langle u^{\prime} u^{\prime}\right\rangle /$ $\partial x$ is larger than $\partial U U / \partial x$ above $50 \mathrm{~m}$.

Because the Reynolds flux divergences are relatively small, when added to the mean circulation momentum flux divergence terms, the total mean momentum flux divergence terms (the three right panels of Fig. 8) retain their general shapes, but with small changes in magnitude and extrema depths. With respect to the EUC, zonal momentum converges meridionally upon the core and divergences vertically away from the core. Zonal momentum diverges zonally within and above the core where the EUC accelerates downstream and converges zonally below the core where the EUC decelerates downstream. Adding these terms together gives the material rate of change of zonal momentum, as shown in
Fig. 9. The divergence of the mean circulation zonal momentum flux $\boldsymbol{\nabla} \cdot \mathbf{V} U$ is positive and maximum within the EUC core and negative near the surface and below the EUC core. The divergence of the Reynolds momentum flux $\boldsymbol{\nabla} \cdot\left\langle\mathbf{v}^{\prime} u^{\prime}\right\rangle$ is positive and maximum near the surface, negative at the EUC core, and relatively small but negative below the EUC core. Upon summation, the total divergence of the mean zonal momentum flux $\boldsymbol{\nabla} \cdot\langle\mathbf{v} u\rangle$ is small near the surface, large and positive within the EUC core, and equally large but negative below the EUC core. This development shows that the recordlength-averaged divergence of the zonal momentum flux at $0^{\circ}, 140^{\circ} \mathrm{W}$ is fully three-dimensional. The contributions by the mean circulation are the largest, but the contributions by the Reynolds fluxes cannot be ignored, particularly between the EUC core and the surface. The magnitude of the $\boldsymbol{\nabla} \cdot\langle\mathbf{v} u\rangle$ profile, of order $3 \times 10^{-7} \mathrm{~m}$ $\mathrm{s}^{-2}$, is comparable to that of the ZPG. Bias, due to the curvature in $U$ is largest at the EUC core. However, this can be quantified (appendix B) and corrected, giving flux divergence scheme results that are nearly identical to those from the advective scheme presented next.

\section{b. Material acceleration by advective formulation}

Using the same format as Fig. 8, Fig. 10 shows the individual terms calculated by the advective scheme. Term by term the advective and flux divergence scheme results are different. The exception is the top row where the zonal derivative terms by the advective scheme are roughly half the magnitude of those by the flux divergence scheme as expected. Zonally, within and above the EUC core fluid accelerates downstream, while below $\nabla \cdot \mathbf{V U}$

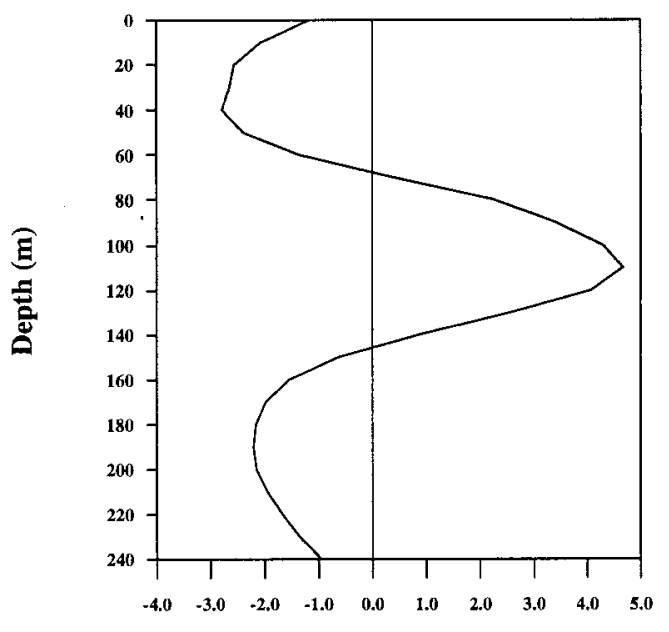

Acceleration $\left(10^{-5} \mathrm{~cm} \mathrm{~s}^{-2}\right)$

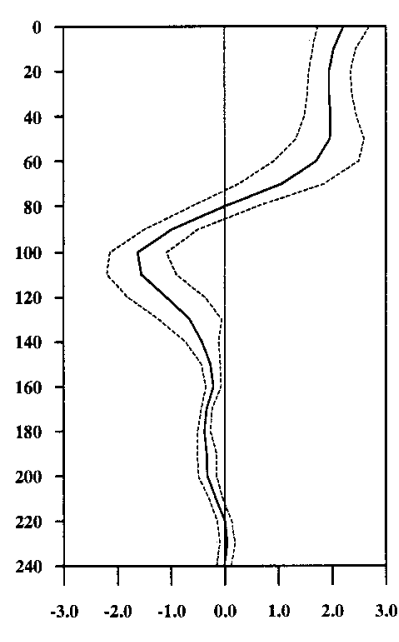

Acceleration $\left(10^{-5} \mathrm{~cm} \mathrm{~s}^{-2}\right)$

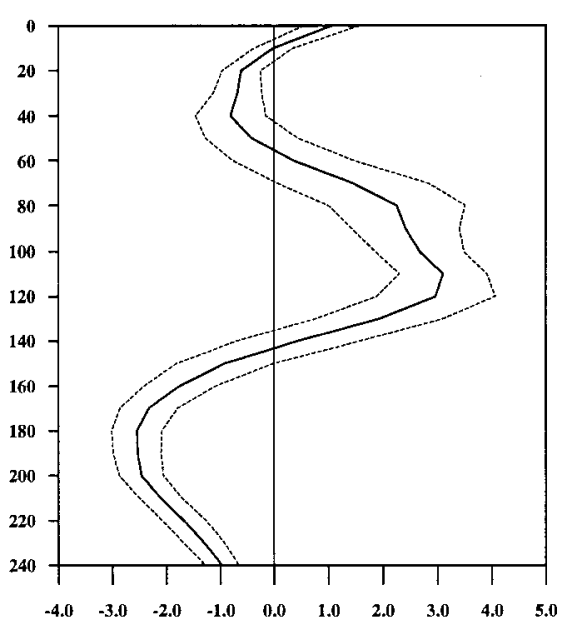

Acceleration $\left(10^{-5} \mathrm{~cm} \mathrm{~s}^{-2}\right)$

FIG. 9. Vertical profiles of the record-length-mean zonal momentum flux divergence by the mean circulation, the resolvable Reynolds stresses, and their sum. The dashed lines represent standard deviations by random fluctuations (appendix A). 
$\mathrm{U} \partial \mathrm{U} / \partial \mathrm{x}$
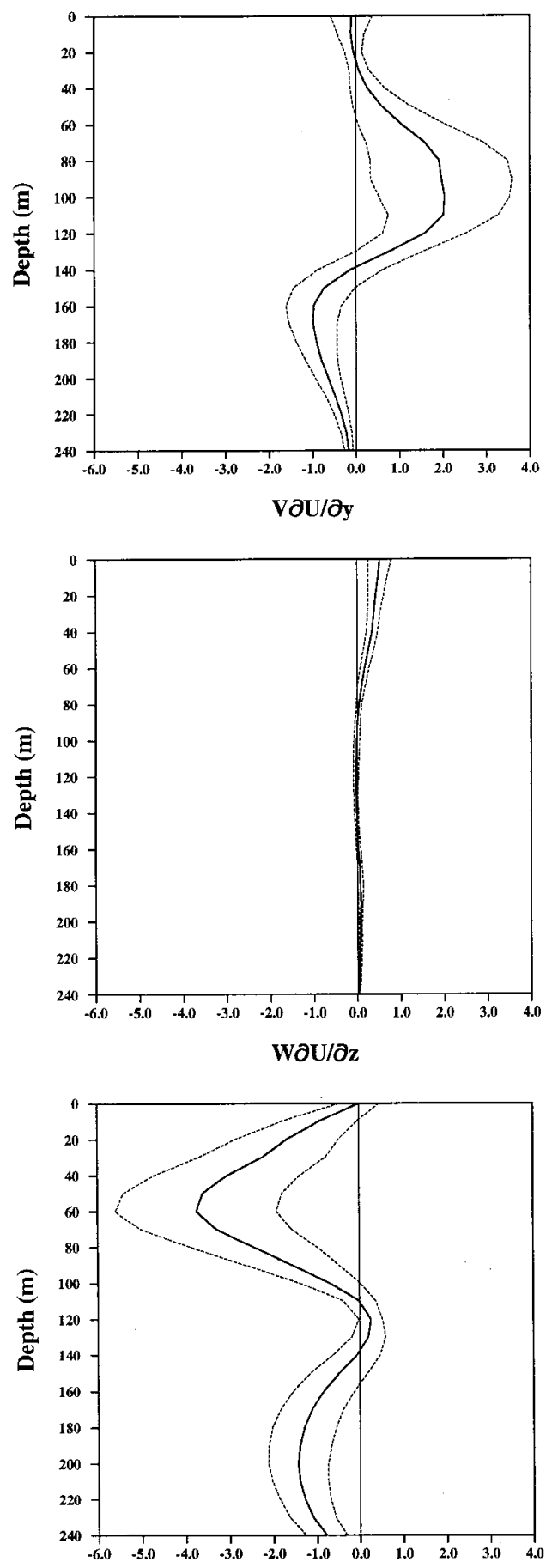

Acceleration $\left(10^{-5} \mathrm{~cm} \mathrm{~s}^{-2}\right)$ $\left\langle\mathbf{u}^{\prime} \partial \mathrm{u}^{\prime} / \partial \mathbf{x}\right\rangle$
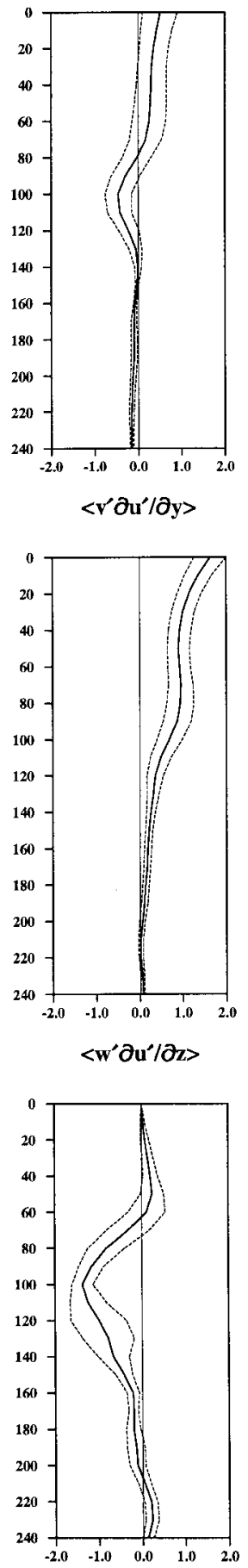

$\langle u \partial u / \partial x>$
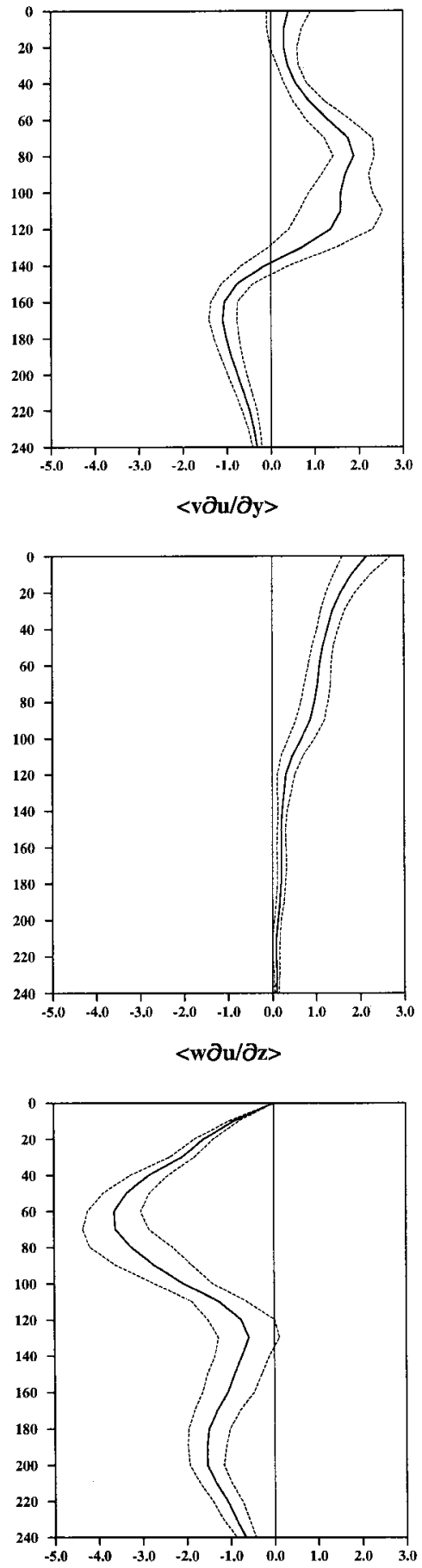

Acceleration $\left(10^{-5} \mathrm{~cm} \mathrm{~s}^{-2}\right)$

FIG. 10. Vertical profiles of the individual constituents making up the record-length mean advection of zonal momentum estimated at $0^{\circ}, 140^{\circ} \mathrm{W}$. The dashed lines represent standard deviations by random fluctuations (appendix A). 
$\mathbf{V} \cdot \nabla \mathbf{U}$

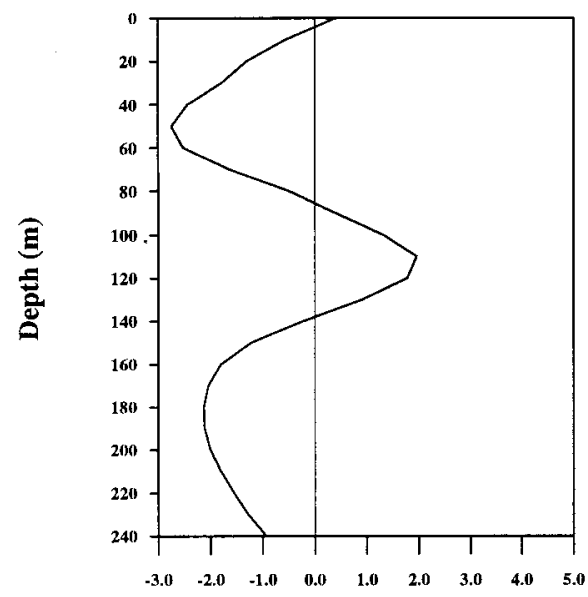

Acceleration $\left(10^{-5} \mathrm{~cm} \mathrm{~s}^{-2}\right)$ $\left\langle\overrightarrow{\mathbf{v}}^{\prime} \cdot \nabla \mathbf{u}^{\prime}\right\rangle$

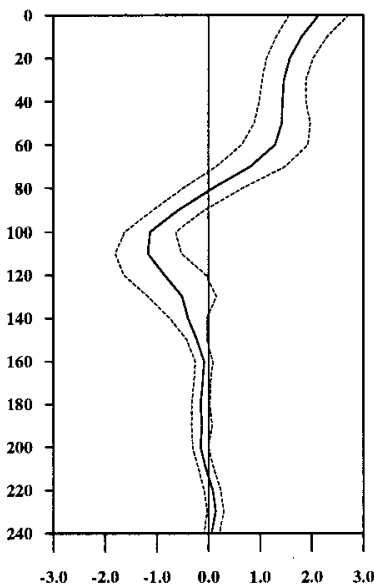

Acceleration $\left(10^{.5} \mathrm{~cm} \mathrm{~s}^{.2}\right)$

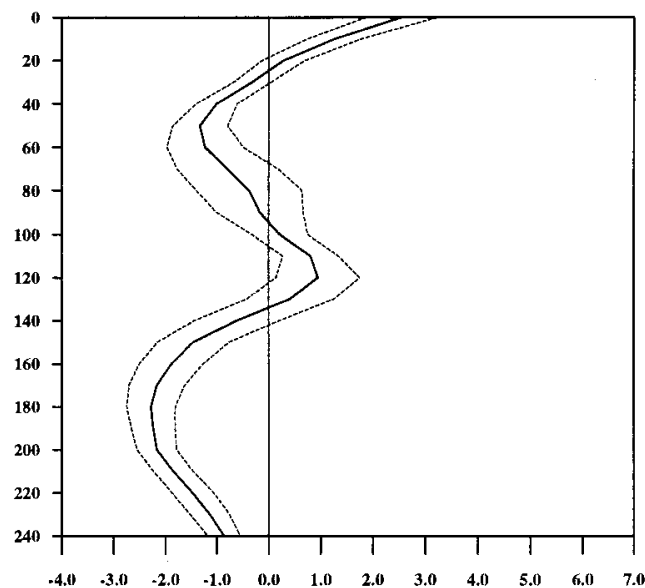

Acceleration $\left(10^{-5} \mathrm{~cm} \mathrm{~s}^{-2}\right)$

FIG. 11. Vertical profiles of the record-length-mean advection of zonal momentum by the mean circulation, the resolvable fluctuations, and their sum. The dashed lines represent standard deviations by random fluctuations (appendix A).

the core fluid decelerates downstream. Vertically, fluid decelerates as it diverges away from the core. However, it is not meaningful to compare the individual terms for these schemes since it is only the summation of all terms that constitutes the material rate of change of zonal momentum. The sum of the three component terms are thus

\section{Pressure Gradient}

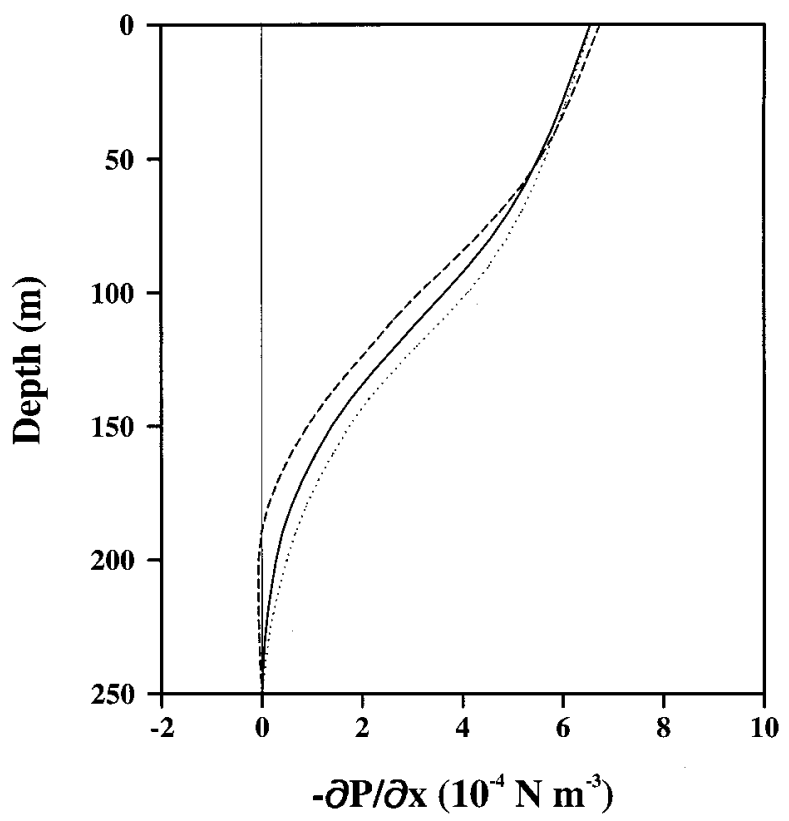

FIG. 12. Vertical profiles of mean zonal pressure gradients estimated between $170^{\circ}$ and $140^{\circ} \mathrm{W}$ (dotted), $140^{\circ}$ and $125^{\circ} \mathrm{W}$ (dashed), and $170^{\circ}$ and $125^{\circ} \mathrm{W}$ (solid) using a reference level of $250 \mathrm{~m}$. shown in Fig. 11. When compared with the flux divergence scheme results, the Reynolds stress contributions are found to be nearly identical. The mean circulation contribution, on the other hand, is smaller within the EUC core since that is where the velocity component curvature is largest. Elsewhere, the two forms are very similar. Upon correcting the flux divergence bias (see appendix B) the discrepancy at the EUC core is accounted for, so consistent results are obtained from the two very different formulations.

From this comparison between zonal-momentum-flux diagnostic schemes it is clear that considering individual terms without the context of their total summation makes little sense because of the three-dimensionality in the material rate of change. It is inappropriate to mix formulations between terms because uninterpretable bias errors result and regardless of formulation the vertical circulation is a critical factor.

\section{c. Zonal pressure gradient and wind stress}

The TOGA-TAO array temperature data on the equator at $125^{\circ} \mathrm{W}, 140^{\circ} \mathrm{W}$, and $170^{\circ} \mathrm{W}$ are used for estimating the ZPG. Salinity is held constant at $35 \mathrm{ppt}$ for lack of data, and using constant salinity versus a historical $T / S$ relationship does not result in significant error (e.g., Weisberg and Weingartner 1986). When referenced to $250 \mathrm{~m}$, the ZPG calculated for the three station pairs are shown in Fig. 12 . For the $125^{\circ}-170^{\circ} \mathrm{W}$ and $140^{\circ}-$ $170^{\circ} \mathrm{W}$ pairs the mean ZPG force is eastward everywhere above $250 \mathrm{~m}$, while for the $125^{\circ}-140^{\circ} \mathrm{W}$ pair it reverses to very small westward values below $180 \mathrm{~m}$. The vertical profiles of the ZPG are consistent with previous measurements (e.g., Mangum and Hayes 1984; 


\section{$\int_{z}^{0} \rho(\mathbf{d u} / \mathbf{d t}, \ldots) \mathbf{d z}$}

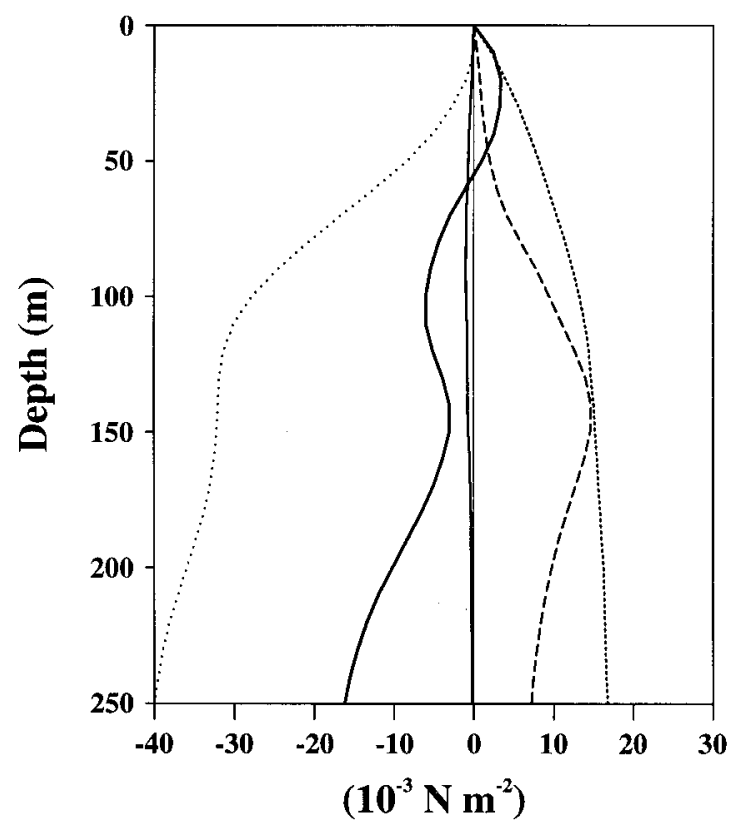

$\int_{z}^{0}-\partial \mathbf{P} / \partial \mathbf{x} \mathbf{d z}$

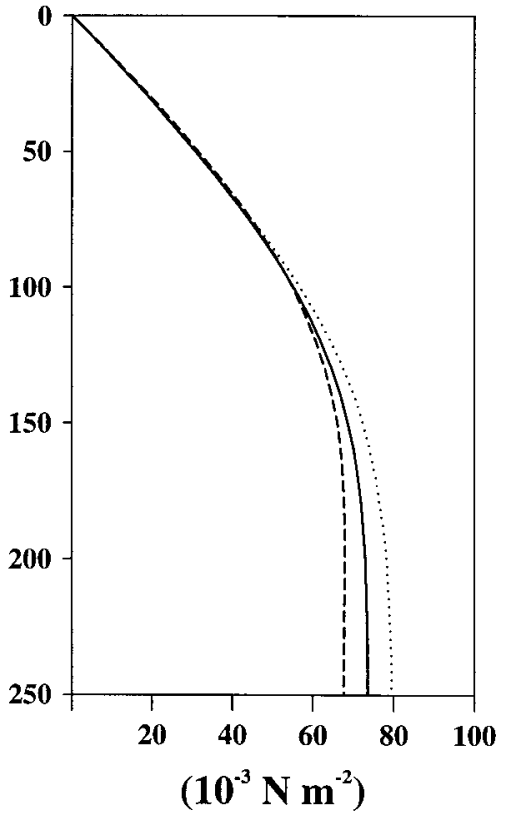

FIG. 13. Mean vertical profiles for vertically integrated constituents of the zonal momentum balance at $0^{\circ}, 140^{\circ} \mathrm{W}$. The left panel includes $\int_{z}^{0} d u / d t d z$ (thick solid); $\int_{z}^{0} \partial u / \partial t d z$ (thin solid); $\int_{z}^{0} u \partial u / \partial x d z$ (long dashed); $\int_{z}^{0} v \partial u / \partial y d z$ (short dashed); and $\int_{z}^{0} w \partial u / \partial z d z$ (dotted). The right panel includes $\int_{z}^{0}-\partial p / \partial x d z$ estimated between $170^{\circ}-140^{\circ} \mathrm{W}$ (dotted), $140^{\circ}-125^{\circ} \mathrm{W}$ (dashed), and $170^{\circ}-125^{\circ} \mathrm{W}$ (solid).

Bryden and Brady 1985; Weisberg and Weingartner 1986; McPhaden and Taft 1988). From Mangum and Hayes (1984), which presents eight individual profiles of the ZPG estimated between $150^{\circ}$ and $110^{\circ} \mathrm{W}$ referenced to $1000 \mathrm{db}$ over the 2-yr period $1979-81$, it is observed that choosing a reference level anywhere between 200 and $1000 \mathrm{db}$ would affect the mean ZPG profile by less than $0.2 \times 10^{-4} \mathrm{~N} \mathrm{~m}^{-3}$. Consequently, choosing the reference level to be $250 \mathrm{~m}$ herein (consistent with Bryden and Brady 1985 and McPhaden and Taft 1988) should not be the limiting factor for the dynamical inferences to be drawn.

Following Large and Pond (1981), and in the same manner as McPhaden and Taft (1988), the zonal component of wind stress is calculated after transforming the buoy winds measured at $4 \mathrm{~m}$ to winds at the standard 10 -m height (under neutrally stable conditions) using a drag coefficient of $1.2 \times 10^{-3}$ and an air density of 1.2 $\mathrm{kg} \mathrm{\textrm {m } ^ { - 3 }}$. The resulting record-length-averaged zonal wind stress component at $0^{\circ}, 140^{\circ} \mathrm{W}$ is $6.1 \times 10^{-2} \mathrm{~N}$ $\mathrm{m}^{-2}$.

\section{d. Vertically integrated zonal momentum balance}

The large, vertically sheared currents on the equator suggest a balance between the ZPG and the vertical stress divergence $\left(\partial \tau^{x} / \partial z\right)$, with any imbalance resulting in a material acceleration $\langle D u / D t\rangle$. To circumvent the problem of unknown $\partial \tau^{x} / \partial z$, a vertically integrated analysis between the surface and any depth $z$ is considered first. The diagnostic equation is

$$
\begin{aligned}
\int_{z}^{0}\langle\mathbf{v} \cdot \boldsymbol{\nabla} u\rangle d z-f \int_{z}^{0} V d z= & -\int_{z}^{0}\left\langle\frac{1}{\rho} \frac{\partial P}{\partial x}\right\rangle d z+\frac{\left\langle\tau_{0}^{x}\right\rangle}{\rho_{0}} \\
& -\left.A_{v} \frac{\partial U}{\partial z}\right|_{z}+\int_{z}^{0} \nabla \cdot\left(A_{h} \nabla U\right) d z
\end{aligned}
$$

where $\left\langle\tau_{0}^{x}\right\rangle$ is the mean zonal wind stress and $\rho_{0}$ is the mean surface density. The Coriolis term is zero on the equator and the horizontal stress divergence is assumed small relative to the vertical stress divergence. It is noted that the horizontal stress divergence is explicitly contained within the estimated Reynolds stress divergence for scales of motion that are resolved. Thus, neglecting the horizontal stress divergence in (7) only neglects effects from unresolved scales. Since the tropical instability waves are the major source of Reynolds stress at all of the five mooring locations, it follows that neglecting the unresolved horizontal stress divergence term here and in subsequent analyses should not be a significant source of error. The integrated $\langle D u / D t\rangle$, the individual terms forming it, and the integrated $\mathrm{ZPG}$ are shown as functions of depth on Fig. 13. The integrated ZPG increases monotonically with depth, reaching the 
$<\partial \tau^{x} / \partial \mathrm{z}>, \rho<\mathrm{Du} / \mathrm{Dt}>$ and $-<\partial \mathrm{P} / \partial \mathrm{x}>$

$\left\langle\tau^{x}\right\rangle$

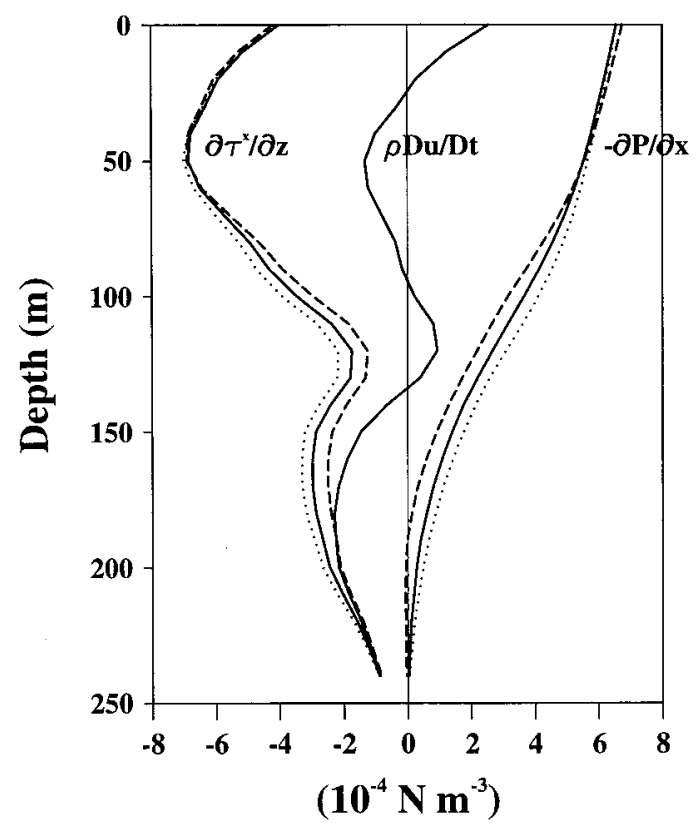

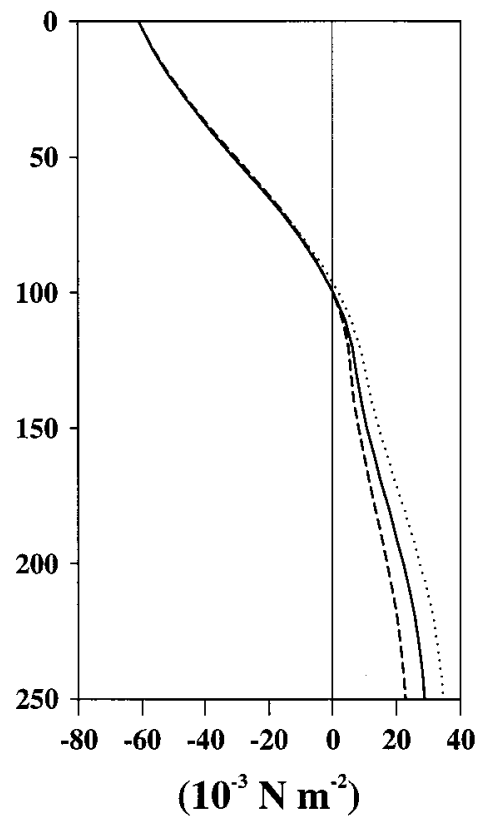

Eddy coefficient $\mathbf{A}_{v}$

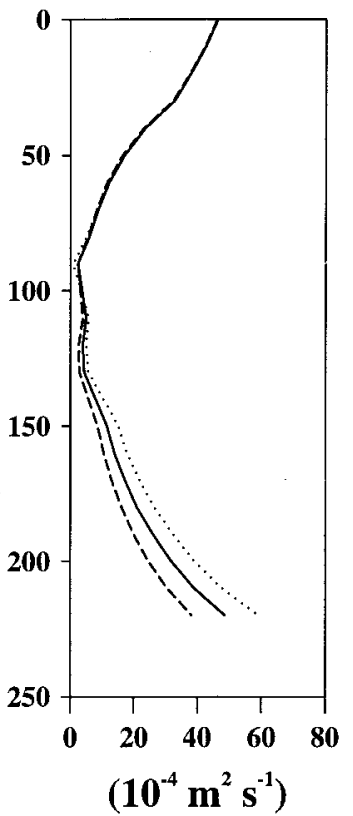

FIG. 14. Mean vertical profiles at $0^{\circ}, 140^{\circ} \mathrm{W}$ of (left panel) the three terms making up the zonal momentum balance where $\left\langle\partial \tau^{x} / \partial z\right\rangle$ is obtained as the residual of the ZPG and $\langle D u / D t\rangle$; (center panel) $\left\langle\tau^{r}\right\rangle$; and (right panel) $A_{v}$. The dotted, dashed, and solid lines represent results using the ZPG estimated between $170^{\circ}-140^{\circ} \mathrm{W}, 140^{\circ}-125^{\circ} \mathrm{W}$, and $170^{\circ}-125^{\circ} \mathrm{W}$, respectively.

estimated value of $\left\langle\tau_{0}^{x}\right\rangle / \rho_{0}$ within about $10 \mathrm{~m}$ of the EUC core (depending upon station pair). Below the core the integrated ZPG attains a nearly constant value. The zonal and meridional terms constituting $\langle D u / D t\rangle$ tend to cancel with the vertical term, so upon integration the acceleration remains small until below the core where it reaches a relatively large negative value. Across the core, roughly between 80 to $160 \mathrm{~m}$, the magnitude of the integrated $\langle D u / D t\rangle$ is relatively constant at less than $10 \%$ the magnitude of $\left\langle\tau_{0}^{x}\right\rangle / \rho_{0}$.

Two important points follow. First, when integrated vertically to the vicinity of the EUC core (located in the thermocline) the momentum balance is linear to within $10 \%$. This explains why linear, reduced-gravity models of the equatorial thermocline's variability in response to $\tau_{0}^{x}$ perform well (e.g., Busalacchi and O'Brien 1981; Weisberg and Tang 1990) despite the presence of nonlinear currents. Second, the relatively small, constant magnitude of the integrated $\langle D u / D t\rangle$ near the EUC core is dynamically consistent with the fact that $\left\langle\tau^{x}\right\rangle / \rho=$ $A_{v} \partial U / \partial z=0$ at the core. From (7), if the integrated ZPG equals $-\left\langle\tau_{0}^{x}\right\rangle / \rho_{0}$ at the vicinity of the core, as observed, then $\left\langle\tau^{x}\right\rangle / \rho$ must equal the integrated $\langle D u / D t\rangle$ (neglecting the horizontal Reynolds stress divergence due to unresolved scales, as argued previously). With $\left\langle\tau^{x}\right\rangle / \rho=$ $A_{v} \partial U / \partial z=0$ at the core, it follows that the integrated $\langle D u / D t\rangle$ should also be zero. Since this occurs within $10 \mathrm{~m}$ of the core, or to within $10 \%$ of $\left\langle\tau_{0}^{x}\right\rangle / \rho_{0}$, we have an independent physical consistency check on the estimation of $\langle D u / D t\rangle$. Along with quantifiable random errors (appendix A), it may now be argued that the finitedifferencing errors are not overwhelming either.

\section{e. The implied vertical stress distribution}

Assuming a balance between the ZPG force, $\rho\langle D u /$ $D t\rangle$, and $\left\langle\partial \tau^{x} / \partial z\right\rangle$, the latter term may be estimated as a residual as shown in Fig. 14. Near the surface the balance is primarily between the eastward directed ZPG force and the westward directed $\left\langle\partial \tau^{x} / \partial z\right\rangle$. This is modified by $\langle D u / D t\rangle$ shaping the near-surface stress divergence. Thus, at $50 \mathrm{~m}$ where $\langle D u / D t\rangle$ has a westward directed maximum because fluid particles decelerate as they move upward away from the EUC core, there must be a corresponding westward directed maximum in vertical friction to account for this deceleration. The increase in $\langle D u / D t\rangle$ from $50 \mathrm{~m}$ to the surface is due to both a decrease in the vertical velocity and positive Reynolds stress (in particular $\left.\left\langle v^{\prime} \partial u^{\prime} / \partial y\right\rangle\right)$ ). By 20-m depth (the mean westward flowing SEC region) the Reynolds stress is sufficient to reverse the sign of $\langle D u / D t\rangle$, thereby accelerating the flow (see Fig. 11) making it less westward downstream. A similar near-surface $\left\langle\partial \tau^{x} / \partial z\right\rangle$ profile was reported by Wacongne (1989) in a numerical circulation model analysis applied to the equatorial Atlantic Ocean and such may be inferred from the Bryden 
and Brady (1985) results. This behavior, resulting from nonlinearity, is now understood in terms of the specific processes that give rise to the observed $\langle D u / D t\rangle$, the underlying cause being vertical advection, but equally important in setting the magnitude and causing a change in direction near the surface is the Reynolds stress.

Below $50 \mathrm{~m}$, as the ZPG force decreases and $\rho\langle D u /$ $D t\rangle$ increases with depth toward the EUC core, these two terms tend to offset resulting in a minimum westward directed $\left\langle\partial \tau^{x} / \partial z\right\rangle$ at the core. Thus, the balance within the EUC core, where the flow accelerates downstream, is weakly nonlinear with the sum of similar magnitude eastward $\rho\langle D u / D t\rangle$ and westward $\left\langle\partial \tau^{x} / \partial z\right\rangle$ balancing the eastward directed ZPG force. Below the EUC core, where the ZPG approaches zero, the balance becomes one between $\rho\langle D u / D t\rangle$ and $\left\langle\partial \tau^{x} / \partial z\right\rangle$. It is within this regime that the EUC decelerates downstream requiring a frictional retarding force in the absence of a sufficiently large westward directed ZPG force.

To summarize, the dynamical flow regimes change in going from the surface through the base of the EUC. The zonal momentum balance goes from one in which the pressure gradient force balances the wind-induced frictional force near the surface (essentially a linear regime modified by nonlinearity), to a weakly nonlinear regime at the EUC core where the pressure gradient force drives a nonlinear acceleration equal in magnitude to the frictional retarding force, to a fully nonlinear regime below the core in which a relative maximum in the frictional retarding force decelerates the EUC downstream.

Integrating the residual-determined $\left\langle\partial \tau^{x} / \partial z\right\rangle$ gives an estimate of the $\left\langle\tau^{x}\right\rangle$ profile, as shown in Fig. 14. Consistent with the summary above, $\left\langle\tau^{x}\right\rangle$ is large, negative, and monotonically increasing from the surface to the EUC core. A zero-crossing occurs within $10 \mathrm{~m}$ of the core, below which $\left\langle\tau^{x}\right\rangle$ is positive (as higher momentum fluid above is rubbing against lower momentum fluid below) and monotonically increasing. This physically required change in sign at the core occurs independent of the $\langle D u / D t\rangle$ estimation, providing some confidence that the errors, both random and systematic, are not controlling. Dividing $\left\langle\tau^{x}\right\rangle$ by $\rho \partial U / \partial z$ provides an estimate of $A_{v}$ (except near the EUC core where $\left\langle\partial \tau^{x} / \partial z\right\rangle$ is divided by $\rho \partial^{2} U / \partial z^{2}$, since the shear is zero), as shown in Fig. 14. This results in values of $40-50\left(\times 10^{-4} \mathrm{~m}^{2}\right.$ $\left.\mathrm{s}^{-1}\right)$ in the near-surface mixed layer decreasing to values around $3 \times 10^{-4} \mathrm{~m}^{2} \mathrm{~s}^{-1}$ within the EUC core and then increasing again below the core. Over the range $150-$ $200 \mathrm{~m}$, where $\langle D u / D t\rangle$ and $\left\langle\partial \tau^{x} / \partial z\right\rangle$ are maximum, $\mathrm{A}_{\mathrm{v}}$ is estimated between $10-20\left(\times 10^{-4} \mathrm{~m}^{2} \mathrm{~s}^{-1}\right)$. It is noted that while the station pairs used for the estimation of the ZPG affects the magnitude of the stress divergence, the stress, and the eddy coefficient estimates of Fig. 14, the different station pairs do not affect the profile shapes or the implications that follow from these.

\section{Temporal evolution of the zonal momentum balance}

Since the relative magnitudes and spatial structures of the SEC and EUC are functions of time, the relative importance of the various terms making up the zonal momentum balance may also be time dependent. Of particular interest are the timescales over which nonlinearity is important. Figure 15 shows the terms making up $D u / D t$ as a function of time and depth, computed by advective formulation using raw hourly data. For presentation, these time series along with others in this section (except the ZPG) have been low-pass filtered to exclude fluctuations at timescales shorter than 10 days. The same calculation using low-pass filtered time series gave nearly identical results, showing that the essential nonlinearities over the spatial scale of the TIWE equatorial array occur over timescales longer than 10 days and that higher-frequency motions do not bias the nonlinear calculations.

The nonlinear acceleration $\mathbf{v} \cdot \nabla u$ is vertically inhomogenous and time dependent. It tends to be positive with largest variability within the EUC core (which migrated between 80 and $150 \mathrm{~m}$ ) and negative above and below the core. Compared to $\mathbf{v} \cdot \boldsymbol{\nabla} u, \partial u / \partial t$ has largest magnitude near the surface and relatively uniform sign with depth. A notable event is the December 1990 eastward momentum pulse. While the local acceleration is well defined and large, the nonlinear acceleration is as large at the EUC core. Such pulses are generally identified as intraseasonal, linear, equatorial Kelvin waves (e.g., Knox and Halpern 1982; McPhaden and Taft 1988) with phase lines traceable across the equatorial Pacific (Kessler et al. 1995). While linearity reasonably describes the vertical integral (Fig. 18), it is found that $\mathbf{v} \cdot \nabla \mathbf{u}$ may be as large as $\partial u / \partial t$ at individual depths.

Of the terms making up $\mathbf{v} \cdot \nabla u, w \partial u / \partial z$ has the largest fluctuations that tend to reverse sign across the EUC core, $u \partial u / \partial x$ tends to be largest in the core, and $v \partial u / \partial y$ tends to be largest near the surface. These are all comparable in magnitude with $\partial u / \partial t$. As with the recordlength average, the time dependence of $\mathbf{v} \cdot \nabla u$ is also fully three-dimensional.

The time dependence of $D u / D t$ should reflect changes in external forcing. Figure 16 shows the ZPG referenced to $250-\mathrm{m}$ depth estimated between $170^{\circ}-140^{\circ} \mathrm{W}, 140^{\circ}-$ $125^{\circ} \mathrm{W}$, and $170^{\circ}-125^{\circ} \mathrm{W}$ as functions of time and depth. The large data gaps and inadequate zonal resolution preclude analyses as performed for the record-length mean, but some qualitative comparisons can be made. The two relative maxima and intervening minimum in the ZPG from July through October 1990 correspond to the relative maxima and intervening minimum in the EUC core speed shown in Fig. 2, and the ZPG maximum in December 1990 corresponds to the Kelvin wave pulse at that time. Thus, the zonal momentum within the EUC responds to changes in the ZPG as expected since an acceleration must occur until $\partial \tau^{x} / \partial z$ adjusts to strike a 


\section{$0^{\circ}, 140^{\circ} \mathrm{W}$}
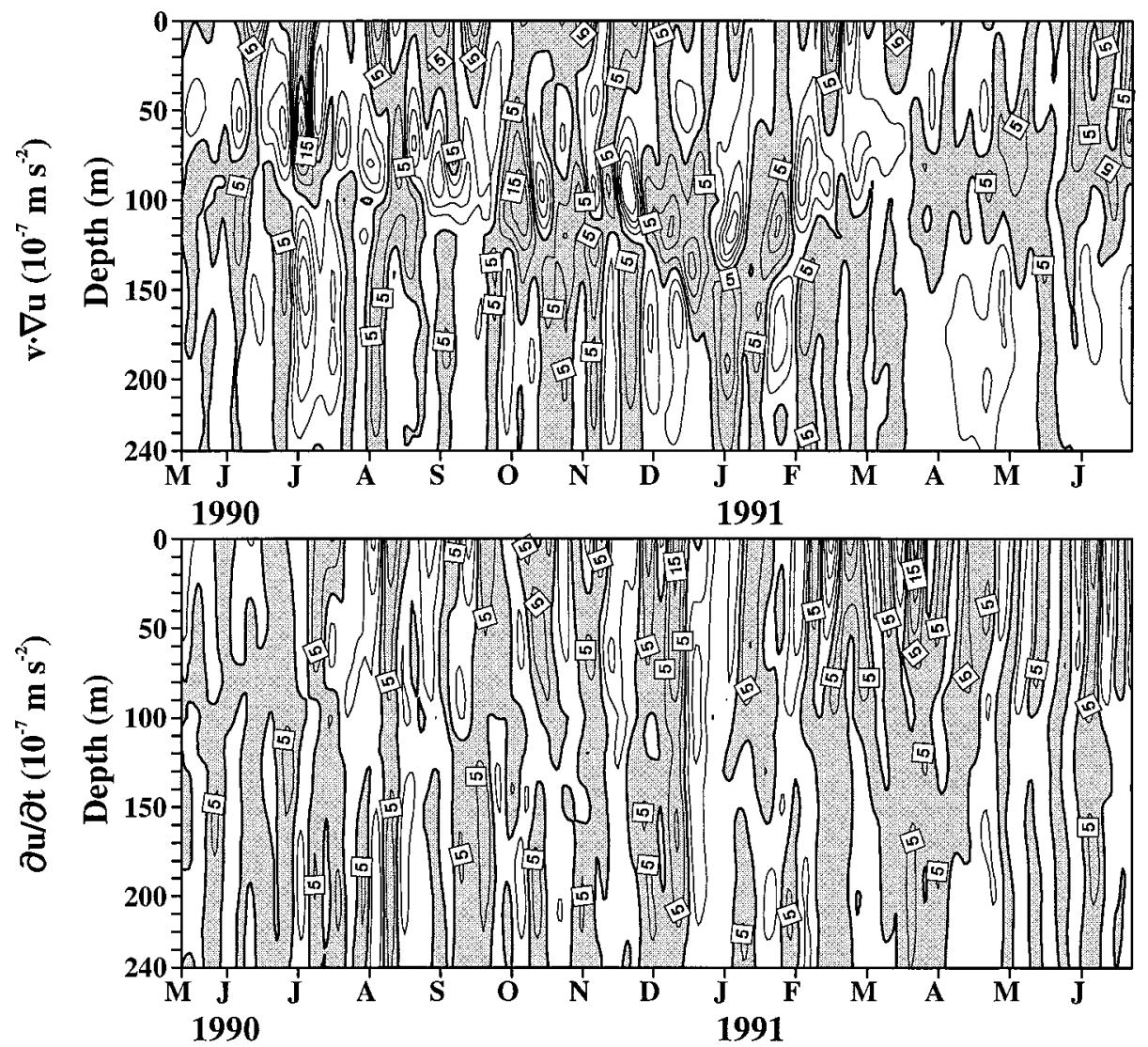

FIG. 15. The nonlinear acceleration $(\mathbf{v} \cdot \nabla u)$, the local acceleration $(\partial u / \partial t)$, and individual constituents making up the nonlinear acceleration $(u \partial u / \partial x, v \partial u / \partial y$, and $w \partial u / \partial z)$ as functions of depth and time. Positive values are stippled, the contour interval is $5 \times 10^{-7} \mathrm{~m} \mathrm{~s}^{-2}$, and all time series have been low-pass filtered to exclude fluctuations on timescales shorter than 10 days.

new balance. However, with a complicated superposition of local (wind stress-see Fig. 19) and remote (pressure gradient) forcing, the time-dependent adjustments of $\partial \tau^{x} / \partial z$ cannot be inferred from the present dataset, primarily due to inadequate ZPG resolution.

Over what frequency range does nonlinearity become important? To address this question, variance density spectra of $\partial u / \partial t$ and $\mathbf{v} \cdot \nabla u$ are shown as a function of frequency and depth in Fig. 17. At frequencies higher than those shown, the $\partial u / \partial t$ spectral densities are at least an order of magnitude larger than those of $\mathbf{v} \cdot \nabla u$. The $\partial u / \partial t(\mathbf{v} \cdot \nabla u)$ spectra decrease (increase) with decreasing frequency. Comparable magnitudes begin to occur in the EUC for frequencies between $0.01 \mathrm{cph}$ and the instability wave frequencies of about $0.002 \mathrm{cph}$. At lower frequencies the $\mathbf{v} \cdot \nabla u$ spectra exceed those of $\partial u / \partial t$, especially in the EUC core. The EUC thus becomes increasingly nonlinear approaching the mean. This is compatible with the finding of Johnson and McPhaden (1993) that the nonlinear interaction between equatorial
Kelvin waves and a three-dimensional mean background circulation field increases with decreasing frequency.

As with the means, the time-dependent $\mathbf{v} \cdot \nabla u$ tend to cancel upon vertical integration. This is shown in Fig. 18 , comparing the integrated $D u / D t, \partial u / \partial t$, and $\mathbf{v} \cdot \nabla u$. The integrated $\mathbf{v} \cdot \boldsymbol{\nabla} u$ is small above the EUC core and then increases in magnitude to maximum values just below the core (at about $150 \mathrm{~m}$ ) before decreasing again. Figure 19 provides a qualitative comparison between the vertically integrated (surface to $250 \mathrm{~m}$ ) $D u / D t, \partial u$ / $\partial t, \mathrm{ZPG}$, and $\tau_{0}^{x}$. The vertically integrated $D u / D t$ and $\partial u /$ $\partial t$ are similar and there is considerable correspondence between these accelerations and the $\tau_{0}^{x}$ fluctuations. Limited correspondence with the ZPG is also evident; particularly for an event in December 1990 attributable to an intraseasonal Kelvin wave that propagated through the array, but since the TOGA-TAO array does not resolve synoptic-scale fluctuations in the ZPG, as observed in the wind and acceleration, the time-varying 


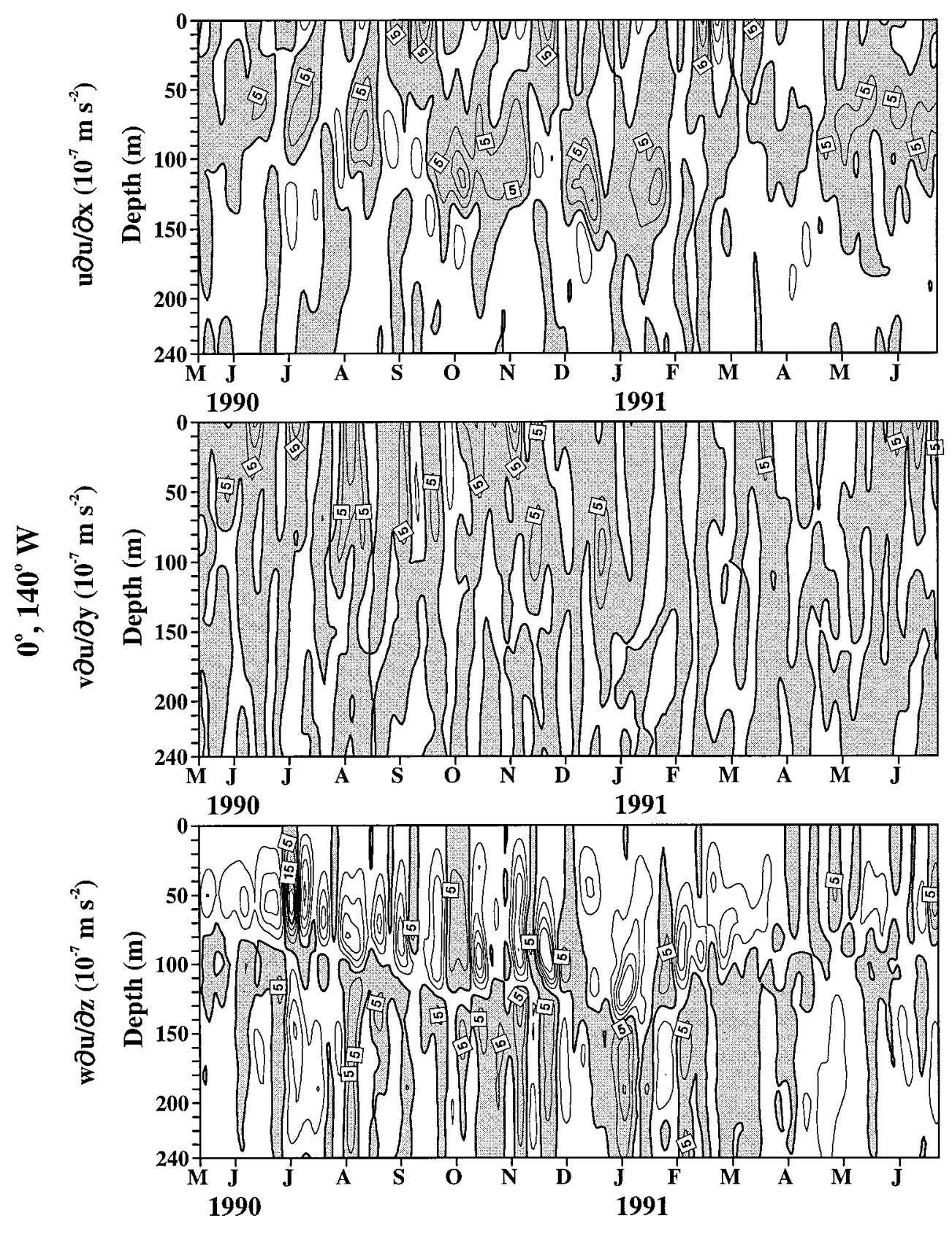

FIG. 15. (Continued)

zonal momentum balance at these scales cannot be diagnosed.

\section{Summary and discussion}

Current velocity data from the TIWE equatorial array have been used to diagnose the upper-ocean zonal momentum balance at $0^{\circ}, 140^{\circ} \mathrm{W}$. The array resolves the large-scale divergence of the upper-ocean currents, thereby permitting an estimation of the zonal momentum flux divergence, the unknown factor in previous empirical studies. Analyses are made of the record- length (13 months) means and fluctuations. In either case the flow is three-dimensional, tending to converge meridionally upon, and diverge vertically away from, the EUC core, consistent with the first comprehensive descriptions of the equatorial circulation by Knauss $(1960,1966)$ and the first theory for the EUC by Fofonoff and Montgomery (1955). The ensuing vertical circulation is found to be a critical element of the zonal momentum balance. The record-length average, dynamical flow regime changes between the surface and the base of the EUC. At the surface it is essentially linear, with the ZPG force balancing the wind-induced fric- 


\section{$-\partial \mathrm{p} / \partial \mathbf{x}\left(10^{-4} \mathrm{~N} \mathrm{~m}^{-3}\right)$}
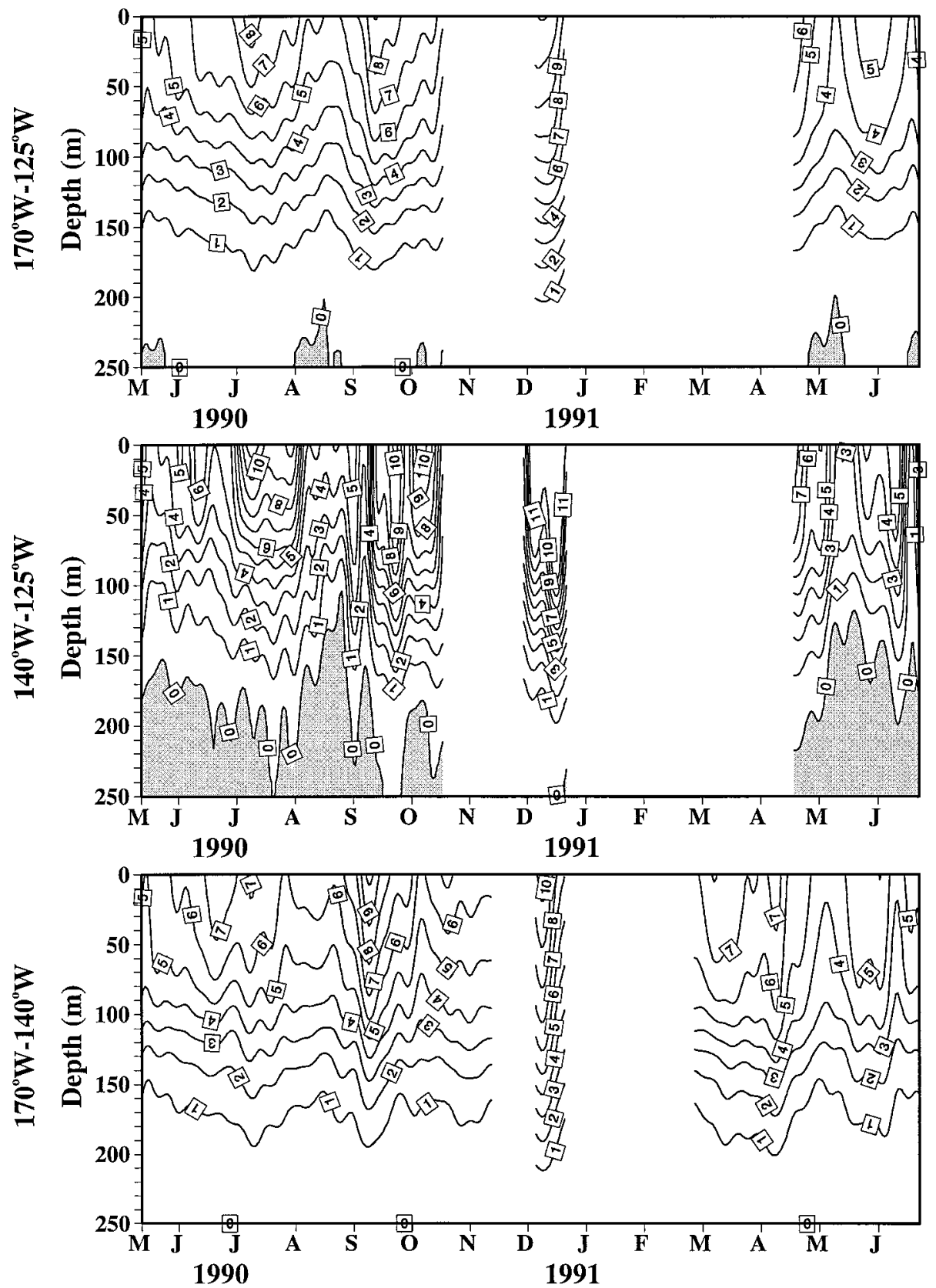

FIG. 16. The ZPG estimated between $170-140^{\circ} \mathrm{W}, 140^{\circ}-125^{\circ} \mathrm{W}$, and $170^{\circ}-125^{\circ} \mathrm{W}$ as functions of depth and time. The small region of westward-directed pressure gradient force is stippled and the contour interval is $10^{-4} \mathrm{~N} \mathrm{~m}^{-3}$.

tional force modified by nonlinearity. At the EUC core it is weakly nonlinear, with the ZPG force driving a nonlinear acceleration equal in magnitude to the frictional retarding force. Below the core (where the ZPG is nominally small or slightly westward) it is fully nonlinear, with the frictional retarding force accounting for the downstream deceleration of the EUC, consistent with the visco-inertial theory of Charney and Spiegel (1971) in which downward vertical advection is necessary to balance friction in the absence of a sufficiently large ZPG.

As with the flow field, the nonlinearities are threedimensional, requiring all three components to characterize the zonal momentum flux divergence. The mean 


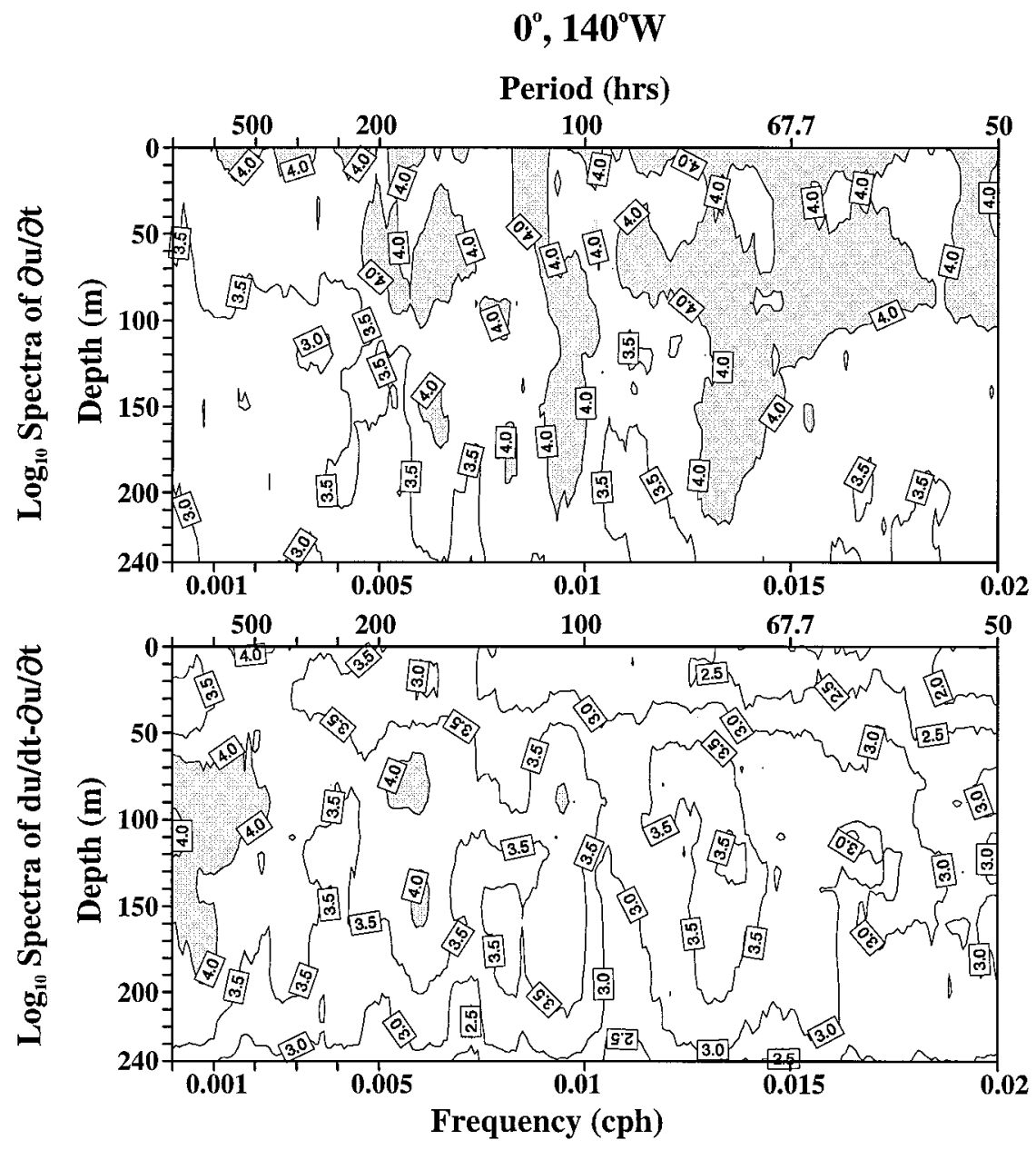

FIG. 17. Log variance densities for $\partial u / \partial t$ and $\mathbf{v} \cdot \nabla u$ as functions of depth and frequency. The contour interval is 0.5 . Light stippling highlights densities greater than $10^{-0.5}\left(\mathrm{~m} \mathrm{~s}^{-2}\right)^{2} \mathrm{cph}^{-1}$. The spectra were averaged over a $0.92 \times 10^{-3} \mathrm{cph}$ bandwidth for approximately 18 degrees of freedom.

zonal momentum flux divergence also contains important Reynolds stress divergences found to occur primarily over the tropical instability wave scales. Their effect in the mean zonal momentum flux divergence is to decelerate downstream both the surface SEC (making it less westward) and the subsurface EUC (making it less eastward), with the horizontal (vertical) Reynolds flux working against the SEC (EUC).

The $\left\langle\partial \tau^{x} / \partial z\right\rangle$ profile is largely affected by the mean zonal momentum flux divergence. The mean vertical advection of eastward momentum combines with the divergence of the horizontal Reynolds fluxes to produce a $\left|\left\langle\partial \tau^{x} / \partial z\right\rangle\right|$ maximum at 50-m depth. Without these nonlinear effects, particularly the mean vertical advection of eastward momentum, $\left|\left\langle\partial \tau^{x} / \partial z\right\rangle\right|$ would be a maximum at the surface as recently found at midlatitude by Chereskin (1995). At the EUC core $\left|\left\langle\partial \tau^{x} / \partial z\right\rangle\right|$ is a minimum owing to the downstream acceleration of the EUC, and it would even be smaller were it not for the resolvable vertical Reynolds flux divergence that reduces the mag- nitude of this acceleration. Below the EUC core $\mid\left\langle\partial \tau^{x}\right|$ $\partial z\rangle \mid$ increases again to a relative maximum necessary to account for the downstream deceleration of the EUC there. The implied vertical profile of $A_{v}$ has near-surface values of $40-50\left(\times 10^{-4} \mathrm{~m}^{2} \mathrm{~s}^{-1}\right)$, decreasing to around $3 \times 10^{-4} \mathrm{~m}^{2} \mathrm{~s}^{-1}$ at the EUC core and increasing again to $10-20\left(\times 10^{-4} \mathrm{~m}^{2} \mathrm{~s}^{-1}\right)$ within the deceleration region below the core. Wilson and Leetmaa (1988) reported a similar profile. The minimum at the core is consistent with the thermocline inhibiting vertical mixing and the estimate agrees with that of Wyrtki and Bennett (1963) arrived at from simple energy considerations using a similar momentum balance.

Quantitatively, the near-surface results for $A_{v}$ agree reasonably well with results from numerous microstructure investigations (e.g., Gregg 1987; Peters et al. 1988; Dillon et al. 1989; Hebert et al. 1991; Lien et al. 1995). However, they differ greatly at and below the EUC core where microstructure measurements suggest values at least an order of magnitude smaller. With the observed 

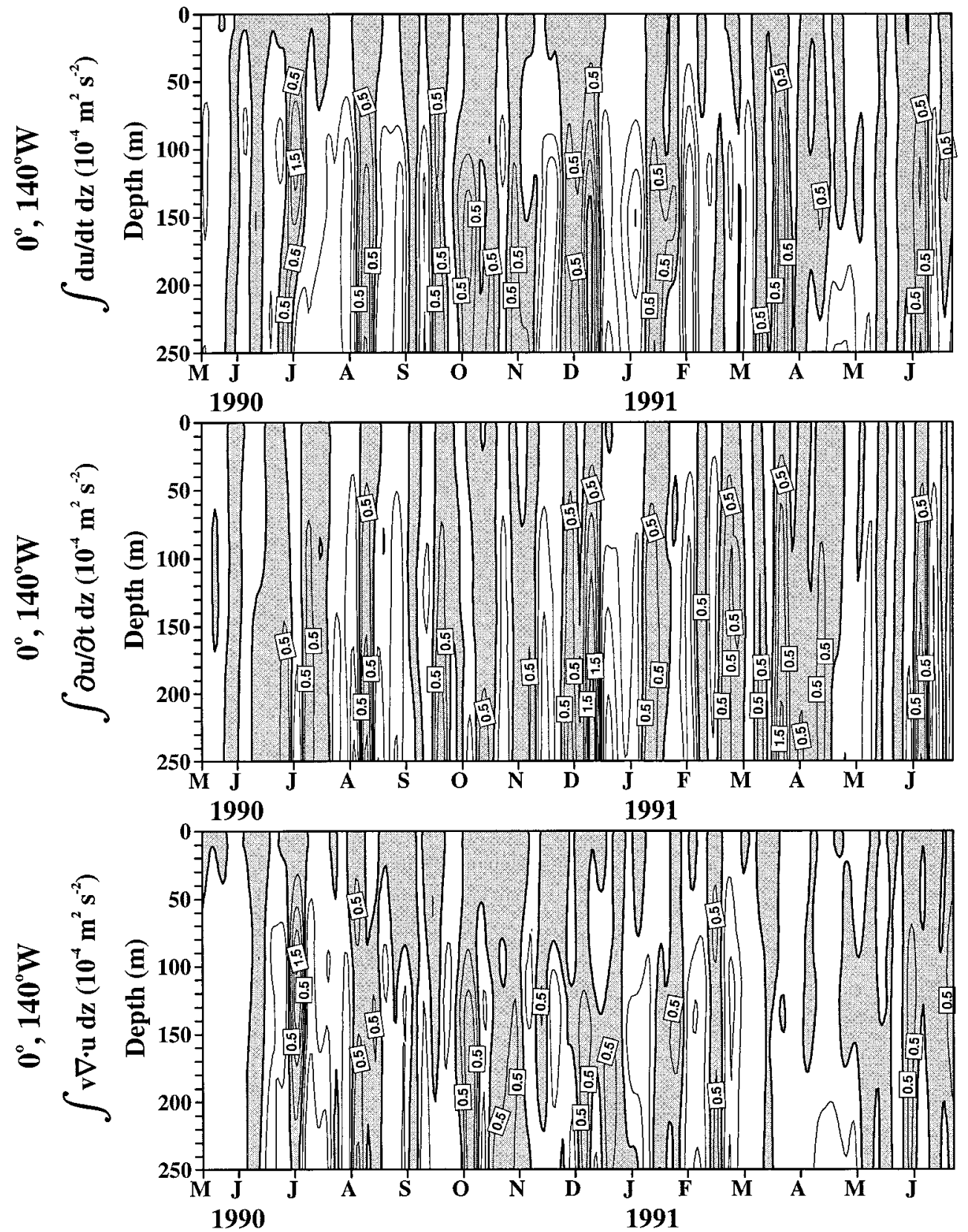

FIG. 18. The vertically integrated zonal acceleration $\left(\int_{z}^{0} D u / D t d z\right)$ and its local $\left(\int_{z}^{0} \partial u / \partial t d z\right)$ and nonlinear $\left(\int_{z}^{0} \mathbf{v} \cdot \nabla u\right.$ $d z$ ) constituents as functions of lower limit of integration and time. Positive values are stippled and the contour interval is $5 \times 10^{-5} \mathrm{~m}^{2} \mathrm{~s}^{-2}$.

downstream deceleration of the EUC below the core demanding a sufficiently large westward directed force, three possible explanations are offered: 1) there exists on average a large $\left(>2 \times 10^{-4} \mathrm{~N} \mathrm{~m}^{-3}\right)$, westward directed $\mathrm{ZPG}$ force below the EUC core; 2) microstructure measurements within the EUC are incorrect; or 3) microstructure measurements do not resolve the scales of motion that produce $\left\langle\partial \tau^{x} / \partial z\right\rangle$ across the EUC. In the absense of observational evidence (e.g., references cited in section $4 \mathrm{c}$ ), the first seems unlikely, and it would also be inconsistent with the observed meridional mass convergence that does not approach zero until the base of the EUC below $230 \mathrm{~m}$. There is no basis herein for discussion of the second, but the third may have merit. Friction ultimately occurs on molecular scale. Eddy friction is just a parameterization for unresolved scales. When resolved, these scales result in quantifiable Reynolds fluxes, which for the present measurements occur on synoptic scales. So, it is possible that the gap between the synoptic and the microstructure scales may contain 

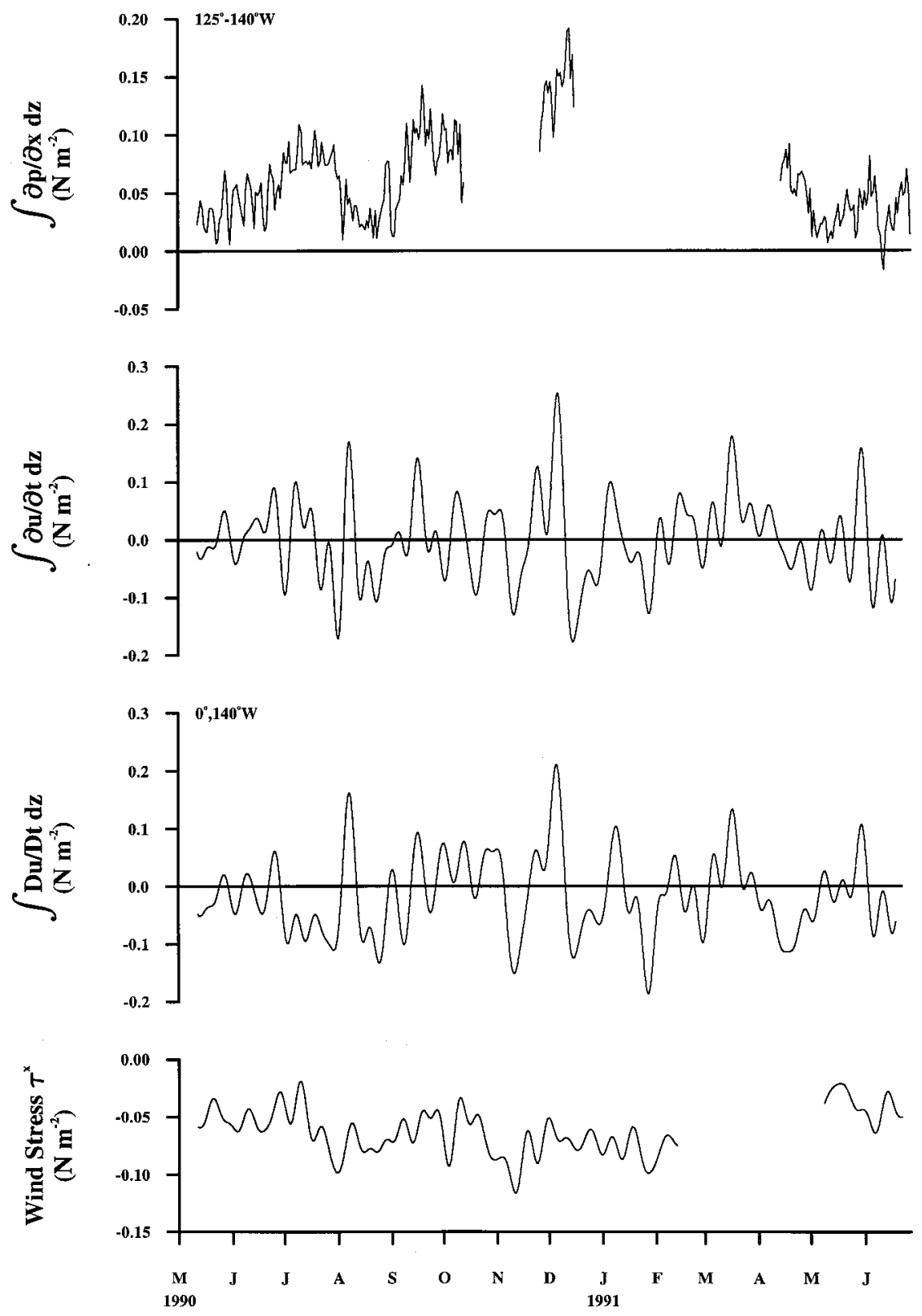

FIG. 19. Time series of the vertically integrated $(0-250 \mathrm{~m}) \mathrm{ZPG}$, local acceleration, total acceleration, and surface zonal wind stress.

other physical processes causing eddy momentum flux divergence. Regardless of such conjecture, something is necessary to decelerate the flow and to account for the mixing of heat (the thermostad) and all other material properties below the EUC core (e.g., Jones 1973; Wyrtki and Kilonsky 1984; although other opinions on thermostad formation have also been offered, e.g., Lukas 1986; McPhaden 1984). In this regard, it is noted that the TIWE dataset gives consistent results for the mixing of heat below the EUC core (Weisberg and Qiao 1996, unpublished manuscript). Also, by not altering the shapes of the curves in Fig. 14, which derive from the $\langle D u / D t\rangle$ estimate, relatively small errors in the independently estimated ZPG or $\tau_{0}^{x}$ do not alter the inferences drawn.

The dynamical inferences drawn for the region below the EUC core are different from the numerical model results of Wacongne (1989) and Yin and Sarachik (1993). In these model cases, however, the magnitude of the frictional force below the EUC core and its partition between horizontal and vertical stress divergences are essentially preset by the subgrid-scale turbulence parameterizations (a constant horizontal eddy diffusivity and a Richardson-number-dependent vertical eddy dif- 
fusivity). The a priori results of such parameterizations are relatively small (when compared with our results) frictional forces below the EUC core and hence no need of a momentum flux divergence by downward vertical advection. This may help explain why the vertical distribution of the vertical velocity component in the models is upwelling everywhere within the EUC, as contrasted with the change from upwelling above and within the EUC core to downwelling below the core calculated with the TIWE equatorial array data. Charney and Spiegel (1971) provide a physical argument for this difference. In their model, with the ZPG and the vertical eddy diffusivity independent of depth, as the eddy diffusivity decreases, the vertical shear and hence the frictional force below the EUC core increases, beyond which it can no longer be balanced by the ZPG force. At this point a downward transport of momentum must develop to balance the frictional force. These findings bring into question the relative roles of the fully threedimensional circulation versus horizontal and vertical eddy diffusivity in maintaining momentum, heat, and other water property balances near the equator. Model studies using smaller horizontal eddy diffusivities and larger background state vertical eddy diffusivities would seem warranted, along with additional empirical studies.

The analyses presented for the time-dependent zonal momentum balance were more qualitative. Ironically, the ZPG (the best determined of the terms in prior studies) is the limiting factor for the time-dependent analysis here. Designed for large-scale monitoring, the TOGATAO array does not resolve the spatial scale of the large amplitude synoptic variability. Consequently, there is a large mismatch in the fluctuations observed in either $\tau_{0}^{x}$ or $D u / D t$ with the fluctuations in the ZPG. TIWE lacked the resources to augment the TOGA-TAO array with closely spaced moorings for temperature and salinity. This was a mistake that future process experiments should avoid. Despite this shortcoming, the large $\tau_{0}^{x}$ and $D u / D t$ fluctuations showed considerable correspondence. Current nonlinearity is largest within the EUC core region, but upon vertical integration the nonlinear terms tend to cancel. Thus, for intraseasonal Kelvin waves, as an example, the local acceleration exceeds the nonlinear effects, especially upon vertical integration. But, nonlinearity increases with decreasing frequency, becoming very important in the mean. This agrees with the observation that the mean meridional scale of the EUC is much less than an equatorial Rossby radius of deformation (the standard deviation of a meridional Gaussian distribution). For example, from Fig. 4 an equivalent meridional scale of about $1^{\circ}$ latitude is calculated, which is smaller by a factor of 3 than an equatorial Rossby radius of deformation $\left[3^{\circ}\right.$ latitude for a Kelvin wave speed of $2.5 \mathrm{~m} \mathrm{~s}^{-1}$, as typically reported (e.g., Kessler et al. 1995)]. Thus, while the intraseasonal fluctuations may be described as nearly linear, the mean EUC is nonlinear. A similar conclusion was found for the equatorial Atlantic by Tang and Weisberg (1993).
In summary, the TIWE equatorial array descriptions of the three-dimensional circulation and zonal momentum flux divergence are consistent with the earliest descriptions and theoretical ideas of the EUC. Estimates of the vertical stress divergence show dynamical flow regimes that change between the surface and the base of the EUC. The vertical stress divergence is much larger over the lower portion of the EUC than previously reported. Nonlinearity becomes increasingly important with decreasing frequency and in the mean the EUC circulation is nonlinear. Nonlinearities tend to cancel upon vertical integration consistent with reduced-gravity models being able to account for equatorial thermocline variations. Future process experiments on the equatorial circulation and heat balance will require higher resolution for the temperature and pressure gradients than provided by the present TOGA-TAO array.

Acknowledgments. Support was provided by the Ocean Sciences Division, National Science Foundation, Grants OCE-8813378 and OCE-9302811. R. Cole and J. Donovan assisted with the field work and analyses, and sea going operations were facilitated by the officers and crew of the R/V Wecoma and Alpha Helix. The TOGA-TAO array wind stress and temperature data were kindly provided by M. McPhaden and L. Mangum of the NOAA/PMEL.

\section{APPENDIX A}

\section{Mean Zonal Momentum Flux Divergence Random Errors}

It is assumed that the measured velocity components are stationary, Gaussian-distributed random variables satisfying the ergodic hypothesis. If their variations result from the superposition of several different physical processes and the record is long enough (has a sufficient number of degrees of freedom) to sample these, then the Gaussian assumption is supported by the central limit theorem.

The $w$ component is estimated by linear operation upon the measured $u$ and $v$ components, so its distribution is also Gaussian. The zonal momentum flux divergence $(\boldsymbol{\nabla} \cdot \mathbf{v} u)$ and its constituents $(\partial u u / \partial x, \partial u v / \partial y$, and $\partial u w / \partial z)$ are estimated by nonlinear operation, so their distributions cannot be specified a priori. However, after forming time series of the velocity component products, their distributions follow the same argument given for the individual velocity components.

The standard deviation for a stationary, Gaussian random variable $x$ is defined as the positive square root of its variance. Let the record-length-averaged estimate of the mean value of $x$ be denoted by $X$ and let the true mean value of $x$ determined by the expectation operator $E\{x\}$ be $\mu_{x}$. The variance of $X$ is thus 
$\operatorname{var}\{X\}=E\left\{\left[X-\mu_{x}\right]^{2}\right\}=E\left\{\left[\frac{1}{T} \int_{0}^{T}\left(x(t)-\mu_{x}\right) d t\right]^{2}\right\}$,

which for large enough $T$ [such that the autocovariance of $x, C_{x}(\tau)$, tends to zero] reduces to (e.g., Bendat and Piersol 1972)

$$
\operatorname{var}\{X\} \approx \frac{1}{T} \int_{-T}^{T} C_{x}(\tau) d \tau
$$

Upon drawing the equivalence between this variance of the mean estimate and the variance of the mean estimate for band-limited noise, this becomes

$$
\operatorname{var}\{X\}=\frac{\tau_{0}}{T} C_{x}(0),
$$

where

$$
\tau_{0}=\frac{\int_{-T}^{T} C_{x}(\tau) d \tau}{C_{x}(0)}
$$

is the integral timescale and $N=T / \tau_{0}$ is the equivalent number of degrees of freedom (e.g., Tennekes and Lumley 1972 or Davis 1977). The significance of the integral timescale is that statistically meaningful estimates of $X$ may be obtained if $T$ is a sufficiently large multiple of $\tau_{0}$, and for $T=N \tau_{0}(N>1)$ the time series may be considered as having $N$ equivalent independent samples. Herein, $\tau_{0}$ was estimated using the smoothed spectrum at zero frequency [since $S(0)=\int_{-T}^{T} C_{x}(\tau) d \tau$ ]. It is noted that sufficiently large $N$ supports the Gaussian assumption via the central limit theorem. In the present dataset only $u$ near the EUC core had $N$ as small as 15 . For all other variables, linear or nonlinear, $N$ was generally larger than 30 .

The analysis of the momentum flux divergence is split into two parts:

$$
\boldsymbol{\nabla} \cdot\langle\mathbf{v} u\rangle=\boldsymbol{\nabla} \cdot \mathbf{v} U+\boldsymbol{\nabla} \cdot\left\langle\mathbf{v}^{\prime} u^{\prime}\right\rangle .
$$

The total and the Reynolds flux terms were analyzed using the above time series approach. With $N$ larger than 30 for all of the nonlinear terms and their sums the Gaussian assumption appears to be satisfactory. Estimating the standard deviation for the first term on the right-hand side of (A1) is more complex. In finite difference form this term may be written as

$$
\boldsymbol{\nabla} \cdot \mathbf{V} U=\frac{\Delta U U}{\Delta x}+\frac{\Delta U V}{\Delta y}+\frac{\Delta U W}{\Delta z} .
$$

Since this term and its constituents are not time series, their random errors must be calculated from the properties of (assumed Gaussian) random variables. For the set of Gaussian random variables $x_{i}(i=1, \cdots, n)$ let the mean, the variance, and the covariance be denoted by
TABLE A1. Covariance of mean velocity pairs used in the standard deviation estimates.

\begin{tabular}{lllllll}
\hline \hline & $U_{i}$ & $V_{i}$ & $W_{i}$ & $U_{j}$ & $V_{j}$ & $W_{j}$ \\
\hline$U_{i}$ & 1 & 0 & 0 & 0.8 & 0 & 0 \\
$V_{i}$ & 0 & 1 & & 0 & 0 & 0.8 \\
$W_{i}$ & 0 & & 1 & 0 & & 0 \\
\hline
\end{tabular}

$$
\begin{aligned}
E\left\{x_{i}\right\} & =\mu_{i} \\
\operatorname{var}\left\{x_{i}\right\} & =\sigma_{i} \\
E\left\{\left(\frac{x_{i}-\mu_{i}}{\sigma_{i}}\right)\left(\frac{x_{j}-\mu_{j}}{\sigma_{j}}\right)\right\} & =C_{i j},
\end{aligned}
$$

respectively. The expected values of products of the $x_{i}$ may then be written as

$$
\begin{aligned}
E\left\{x_{i} x_{j}\right\}= & C_{i j} \sigma_{i} \sigma_{j}+\mu_{i} \mu_{j} \\
E\left\{x_{i} x_{j} x_{k}\right\}= & \mu_{k} \sigma_{i} \sigma_{j} C_{i j}+\mu_{j} \sigma_{i} \sigma_{k} C_{i k}+\mu_{i} \sigma_{j} \sigma_{k} C_{j k}+\mu_{i} \mu_{j} \mu_{k} \\
E\left\{x_{i} x_{j} x_{k} x_{l}\right\}= & \sigma_{i} \sigma_{j} \sigma_{k} \sigma_{l}\left[C_{i j} C_{k l}+C_{i k} C_{j l}+C_{i l} C_{j k}\right] \\
& +\mu_{k} \mu_{l} \sigma_{i} \sigma_{j} C_{i j}+\mu_{j} \mu_{l} \sigma_{i} \sigma_{k} C_{i k}+\mu_{j} \mu_{k} \sigma_{i} \sigma_{l} C_{i l} \\
& +\mu_{i} \mu_{l} \sigma_{j} \sigma_{k} C_{j k}+\mu_{i} \mu_{k} \sigma_{j} \sigma_{l} C_{j l}+\mu_{i} \mu_{j} \sigma_{k} \sigma_{l} C_{k l} \\
& +\mu_{i} \mu_{j} \mu_{k} \mu_{l} .
\end{aligned}
$$

These allow the variances of the finite difference terms to be expressed as

$$
\begin{aligned}
\operatorname{var}\left\{x_{1} x_{2}-x_{3} x_{4}\right\}= & E\left\{\left[x_{1} x_{2}-x_{3} x_{4}\right]^{2}\right\}-E^{2}\left\{\left[x_{1} x_{2}-x_{3} x_{4}\right]\right\} \\
= & \sigma_{1}^{2} \sigma_{2}^{2}\left[C_{12}^{2}+1\right]+2 \mu_{1} \mu_{2} \sigma_{1} \sigma_{2} C_{12}+\mu_{1}^{2} \sigma_{2}^{2} \\
& +\mu_{2}^{2} \sigma_{1}^{2}+\sigma_{3}^{2} \sigma_{4}^{2}\left[C_{34}^{2}+1\right] \\
& +2 \mu_{3} \mu_{4} \sigma_{3} \sigma_{4} C_{34}+\mu_{3}^{2} \sigma_{4}^{2}+\mu_{4}^{2} \sigma_{3}^{2} \\
& -2 \sigma_{1} \sigma_{2} \sigma_{3} \sigma_{4}\left[C_{13} C_{24}+C_{14} C_{23}\right] \\
& -2 \mu_{2} \mu_{4} \sigma_{1} \sigma_{3} C_{13}-2 \mu_{2} \mu_{3} \sigma_{1} \sigma_{4} C_{14} \\
& -2 \mu_{1} \mu_{4} \sigma_{2} \sigma_{3} C_{23}-2 \mu_{1} \mu_{3} \sigma_{2} \sigma_{4} C_{24}
\end{aligned}
$$

The standard deviation estimates for $\Delta U U / \Delta x, \Delta U V / \Delta y$, and $\Delta U W / \Delta z$ then follow by replacing the variables $x_{i}$ in (A3) with the appropriate $U, V$, and $W$ and then multiplying by a factor $1 / \Delta x^{2}, 1 / \Delta y^{2}$, or $1 / \Delta z^{2}$.

The covariance values $C_{i j}$ used in the estimations are shown in Table A1. These were obtained from estimates of coherence at zero frequency (by averaging over the lowest-frequency portion of the spectrum) with the understanding that the superposition of variations having mixed symmetry properties about the equator will decrease covariances between component pairs at different locations. Thus, high covariance between $U$ pairs and $W$ pairs are obtained, compared with lower coherence between off-equator $V$ pairs. With no consistent set of values to choose for lower coherence pairs, these were set to zero in the standard deviation estimation. Since 
the small, omitted terms have different signs in (A3), they tend to cancel so the net effect of this omission is relatively small. With this technique the standard deviations for each of the individual terms on the righthand side of (A2) were estimated. The standard deviation of the sum was not. As a divergence, the errors should tend to cancel like the terms making up the sum, but there is no way of objectively determining this. Simply adding the variances of each term together would provide a meaningless overestimate of the standard deviation for the divergence.

A similar development using the expected values of products of the $x_{i}$ was employed for the standard deviation analysis of the advective scheme terms.

\section{APPENDIX B}

\section{Advective and Flux Divergence Scheme Comparison}

The central finite differences for the individual terms in advective and flux divergence schemes, respectively, are

$$
\begin{aligned}
& u \frac{\partial u}{\partial x} \approx u_{4} \frac{u_{3}-u_{1}}{x_{3}-x_{1}} \\
& v \frac{\partial u}{\partial y} \approx v_{4} \frac{u_{5}-u_{2}}{y_{5}-y_{2}} \\
& w \frac{\partial u}{\partial z} \approx \bar{w} \frac{u_{u}-u_{1}}{\Delta z}
\end{aligned}
$$

and

$$
\begin{aligned}
& \frac{\partial u u}{\partial x} \approx \frac{u_{3} u_{3}-u_{1} u_{1}}{x_{3}-x_{1}}=\frac{u_{3}+u_{1}}{2} \frac{u_{3}-u_{1}}{x_{3}-x_{1}}+\frac{u_{3}+u_{1}}{2} \frac{u_{3}-u_{1}}{x_{3}-x_{1}} \\
& \frac{\partial u v}{\partial y} \approx \frac{u_{5} v_{5}-u_{2} v_{2}}{y_{5}-y_{2}}=\frac{v_{5}+v_{2}}{2} \frac{u_{5}-u_{2}}{y_{5}-y_{2}}+\frac{u_{5}+u_{2}}{2} \frac{v_{5}-v_{2}}{y_{5}-y_{2}} \\
& \frac{\partial u w}{\partial z} \approx \frac{u_{u} w_{u}-u_{1} w_{1}}{\Delta z}=\bar{w} \frac{u_{u}-u_{1}}{\Delta z}+\frac{u_{u}+u_{1}}{2} \frac{w_{u}-w_{1}}{\Delta z},
\end{aligned}
$$

where the subscripts are either station numbers or the upper and lower layers, respectively; $\bar{w}$ is the average $w$ between upper and lower layers and $\Delta z$ is the $10-\mathrm{m}$ layer spacing. In differential form, these two schemes are equivalent via continuity. Is this true of the finitedifference forms, or is one biased relative to the other? Note that with small acceptable error the central values $u_{4}$ and $v_{4}$ may be approximated by averages of their horizontally adjacent values

$$
u_{4} \approx \frac{u_{3}+u_{1}}{2}, \quad v_{4} \approx \frac{v_{5}+v_{2}}{2} .
$$

This approximation allows the flux divergence form to be rewritten as

$$
\begin{aligned}
\frac{\Delta u u}{\Delta x}+\frac{\Delta u v}{\Delta y}+\frac{\Delta u w}{\Delta z} \approx u \frac{\Delta u}{\Delta x} & +v \frac{\Delta u}{\Delta y}+w \frac{\Delta u}{\Delta z} \\
+ & \frac{\left(u_{3}+u_{1}\right.}{2} \frac{u_{3}-u_{1}}{x_{3}-x_{1}} \\
& +\frac{u_{5}+u_{2}}{2} \frac{v_{5}-v_{2}}{y_{5}-y_{2}} \\
& \left.+\frac{u_{u}+u_{1}}{2} \frac{w_{u}-w_{1}}{\Delta z}\right) .
\end{aligned}
$$

Thus, an equivalency is achieved between the flux divergence and the advective schemes if the term in parentheses on the right-hand side is zero. This occurs by continuity if

$$
\frac{u_{3}+u_{1}}{2}, \quad \frac{u_{u}+u_{1}}{2}, \text { and } \frac{u_{5}+u_{2}}{2}
$$

are equal. The mean velocity component profiles of Fig. 4 show that $\left(u_{3}+u_{1}\right) / 2$ and $\left(u_{u}+u_{1}\right) / 2$ are both approximately equal to $u_{4}$; however, $\left(u_{5}+u_{2}\right) / 2$ is generally less than $\mathrm{u}_{4}$. This discrepancy is largest within the EUC core where $\left(u_{5}+u_{2}\right) / 2$ is about $u_{4} / 2$. Thus, near the core

$$
\begin{aligned}
& \frac{u_{3}+u_{1}}{2} \frac{u_{3}-u_{1}}{x_{3}-x_{1}}+\frac{u_{5}+u_{2}}{2} \frac{v_{5}-v_{2}}{y_{5}-y_{2}}+\frac{u_{u}+u_{1}}{2} \frac{w_{u}-w_{1}}{\Delta z} \\
& \approx u_{4}\left(\frac{u_{3}-u_{1}}{x_{3}-x_{1}}+\frac{v_{5}-v_{2}}{y_{5}-y_{2}}+\frac{w_{u}-w_{1}}{\Delta z}\right)-\frac{u_{4}}{2} \frac{v_{5}-v_{2}}{y_{5}-y_{2}} \\
& =-\frac{u_{4}}{2} \frac{v_{5}-v_{2}}{y_{5}-y_{2}},
\end{aligned}
$$

where the continuity equation has been applied. Therefore, the flux divergence scheme is biased relative to the advective scheme, with the bias

$$
-\frac{u_{4}}{2} \frac{v_{5}-v_{2}}{y_{5}-y_{2}}
$$

being due to the meridional curvature of the $u$ component causing $\left(u_{5}+u_{2}\right) / 2$ to be different from $u_{4}$. Physically, the flux divergence formulation fails to conserve mass; but, a posteriori, this is a quantifiable, correctable error so that inferences drawn from the flux divergence scheme remain valuable. For the TIWE equatorial array data such bias applies primarily to the mean circulation. For the Reynolds fluxes, in contrast to the mean, the results from the two different schemes are nearly identical, since the meridional curvature for the fluctuations is small relative to the mean and

$$
\frac{u_{5}^{\prime}+u_{2}^{\prime}}{2} \approx u_{4}^{\prime} \text {. }
$$

\section{REFERENCES}

Arthur, R. S., 1960: A review of the calculation of ocean currents at the equator. Deep-Sea Res., 6, 287-297. 
Bendat, J. S., and A. G. Piersol, 1972: Random Data. Wiley-Interscience, $407 \mathrm{pp}$.

Bryden, H. L., and E. C. Brady, 1985: Diagnostic model of threedimensional circulation in the upper equatorial Pacific Ocean. $J$. Phys. Oceanogr., 15, 1255-1273.

$\longrightarrow$, and — 1989: Eddy momentum and heat fluxes and their effects on the circulation of the equatorial Pacific Ocean. J. Mar. Res., 47, 55-79.

Busalacchi, A. T., and J. J. O'Brien, 1981: Interannual variability of the equatorial Pacific in the 1960s. J. Geophys. Res., 86, 10 90110907 .

Charney, J. G., 1960: Non-linear theory of a wind-driven homogeneous layer near the equator. Deep-Sea Res., 6, 303-310.

- - and S. Spiegel, 1971: Structure of wind driven equatorial currents in homogeneous oceans. J. Phys. Oceanogr., 1, 149-160.

Chereskin, T. K., 1995: Direct evidence for an Ekman balance in the California Current. J. Geophys. Res., 100, 18 261-18 269.

Cromwell, T., R. B. Montgomery, and E. D. Stroup, 1954: Equatorial under-current in Pacific Ocean revealed by new methods. Science, 119, 648-649.

Davis, R. E., 1977: Techniques for statistical analysis and prediction of geophysical fluid systems. Geophys. Astrophys. Fluid Dyn. 8, 245-277.

Dillon, T. M., J. N. Moum, T. K. Chereskin, and D. R. Caldwell, 1989: Zonal momentum balance at the Equator. J. Phys. Oceanogr., 19, 561-570.

Fofonoff, N. P., and R. B. Montgomery, 1955: The Equatorial Undercurrent in the light of the vorticity equation. Tellus, 7, 518521.

Gregg, M. C., 1987: Diapycnal mixing in the thermocline: A review. J. Geophys. Res., 92, 5249-5286.

Hansen, D., and C. Paul, 1984: Genesis and effects of long waves in the equatorial Pacific. J. Geophys. Res., 89, $10431-10440$.

Hebert, D., J. N. Moum, C. A. Paulson, D. R. Caldwell, T. K. Chereskin, and M. J. McPhaden, 1991: The role of the turbulent stress divergence in the equatorial Pacific zonal momentum balance. J. Geophys. Res., 96, 7127-7136.

Johnson, E. S., and M. J. McPhaden, 1993: Structure of intraseasonal Kelvin waves in the equatorial Pacific Ocean. J. Phys. Oceanogr., 23, 608-625.

, and D. S. Luther, 1994: Mean zonal momentum balance in the upper and central equatorial Pacific Ocean. J. Geophys. Res., 99, 7689-7705.

Jones, J. H., 1973: Vertical mixing in the Equatorial Undercurrent. J. Phys. Oceanogr., 3, 286-296.

Kessler, W. S., M. J. McPhaden, and K. M. Weickmann, 1995: Forcing of intraseasonal Kelvin waves in the equatorial Pacific. J. Geophys. Res., 100, $10613-10632$

Knauss, J. A., 1960: Measurements of the Cromwell Current. DeepSea Res., 6, 265-286.

, 1966: Further measurements and observations of the Cromwell Current. J. Mar. Res., 24, 205-240.

Knox, R. A., and D. Halpern, 1982: Long range Kelvin wave propagation of transport variations in Pacific Ocean equatorial currents. J. Mar. Res., 40(Suppl.), 329-339.

Large, W. G., and S. Pond, 1981: Open ocean momentum flux measurements in moderate to strong winds. J. Phys. Oceanogr., 11, 324-336.

Lien, R.-C., D. R. Caldwell, M. C. Greg, and J. N. Moum, 1995:
Turbulence variability at the equator in the central Pacific at the begining of the 1991-1993 El Niño. J. Geophys. Res., 100, 68816898.

Lukas, R., 1986: The termination of the Equatorial Undercurrent in the eastern Pacific. Progress in Oceanography, Vol. 16, Pergamon Press, 63-90.

- 1987: Horizontal Reynolds stresses in the central equatorial Pacific. J. Geophys. Res., 92, 9453-9463.

Luther, D. S., and E. S. Johnson, 1990: Eddy energetics in the upper equatorial Pacific during the Hawaii-to-Tahiti shuttle experiment. J. Phys. Oceanogr., 20, 913-944.

Mangum, L. J., and S. P. Hayes, 1984: The vertical structure of the zonal pressure gradient in the eastern equatorial Pacific. J. Geophys. Res., 89, $10441-10450$.

McPhaden, M. J., 1984: On the dynamics of equatorial subsurface countercurrents. J. Phys. Oceanogr., 14, 1216-1225.

— variability in the eastern equatorial Pacific. J. Phys. Oceanogr., 18, 1713-1732.

Peters, H., M. C. Gregg, and J. M. Toole, 1988: On the parameterization of equatorial turbulence. J. Geophys. Res., 93, 11991218.

Qiao, L., and R. H. Weisberg, 1995: Tropical instability wave kinematics: Observations from the Tropical Instability Wave Experiment. J. Geophys. Res., 100, 8677-8693.

Stommel, H., 1960: Wind-drift near the Equator. Deep-Sea Res., 6, 298-302.

Tang, T. Y., and R. H. Weisberg, 1993: Seasonal variations in equatorial Atlantic Ocean zonal volume transport at $28^{\circ} \mathrm{W}$. J. Geophys. Res., 98, $10145-10153$.

Tennekes, H., and J. L. Lumley, 1972: A First Course in Turbulence. The MIT Press, 300 pp.

Wacongne, S., 1989: Dynamical regimes of a fully nonlinear stratified model of the Atlantic Equatiorial Undercurrent. J. Geophys. Res., 94, 4801-4815.

Weisberg, R. H., and T. J. Weingartner, 1986: On the baroclinic response of the zonal pressure gradient in the equatorial Atlantic Ocean. J. Geophys. Res., 91, 11 717-11 725.

- , and - 1988: Instability waves in the equatorial Atlantic Ocean. J. Phys. Oceanogr., 18, 1641-1657.

- , and T. Y. Tang, 1990: A linear analysis of equatorial Atlantic Ocean thermocline variability. J. Phys. Oceanogr., 20, 18131825.

- J. C. Donovan, and R. D. Cole, 1991: The Tropical Instability Wave Experiment (TIWE) Equatorial Array: A report on data collected using subsurface moored acoustic Doppler current profilers, May 1990-June 1991. Tech. Rep., 84 pp. [Available from Dept. of Marine Science, University of South Florida, 140 Seventh Ave., St. Petersburg, FL 33701.]

Wilson, D., and A. Leetmaa, 1988: Acoustic Doppler current profiling in the equatorial Pacific in 1984. J. Geophys. Res., 93, 13 94713966.

Wyrtki, K., and E. B. Bennett, 1963: Vertical eddy viscosity in the Pacific Equatorial Undercurrent. Deep-Sea Res., 10, 449-455. and B. Kilonsky, 1984: Mean water and current structure during the Hawaii-to-Tahiti Shuttle Experiment. J. Phys. Oceanogr., 14, 242-254.

Yin, F. L., and E. S. Sarachik, 1993: Dynamics and heat balance of steady equatorial undercurrents. J. Phys. Oceanogr., 23, 16471669. 